\title{
Langmuir Mixing Effects on Global Climate: WAVEWATCH III in CESM
}

\author{
Qing $\mathrm{Li}^{\mathrm{a}, *}$, Adrean $\mathrm{Webb}^{\mathrm{b}}$, Baylor Fox-Kemper ${ }^{\mathrm{a}}$, Anthony Craig ${ }^{\mathrm{c}}$, Gokhan \\ Danabasoglu ${ }^{c}$, William G. Large ${ }^{c}$, Mariana Vertenstein ${ }^{c}$ \\ ${ }^{a}$ Dept. of Earth, Environmental and Planetary Sciences, Brown University, Providence, \\ Rhode Island, USA \\ ${ }^{b}$ Dept. of Ocean Technology, Policy, and Environment, The University of Tokyo, Kashiwa, \\ Chiba, Japan \\ ${ }^{c}$ National Center for Atmospheric Research, Boulder, Colorado, USA
}

\begin{abstract}
Large-Eddy Simulations (LES) have shown the effects of ocean surface gravity waves in enhancing the ocean boundary layer mixing through Langmuir turbulence. Neglecting this Langmuir mixing process may contribute to the common shallow bias in mixed layer depth in regions of the Southern Ocean and the Northern Atlantic in most state-of-the-art climate models. In this study, a third generation wave model, WAVEWATCH III, has been incorporated as a component of the Community Earth System Model, version 1.2 (CESM1.2). In particular, the wave model is now coupled with the ocean model through a modified version of the K-Profile Parameterization (KPP) to approximate the influence of Langmuir mixing. Unlike past studies, the wind-wave misalignment and the effects of Stokes drift penetration depth are considered through empirical scalings based on the rate of mixing in LES. Wave-Ocean only experiments show substantial improvements in the shallow biases of mixed layer depth in the Southern Ocean. Ventilation is enhanced and low concentration biases of pCFC-11 are reduced in the Southern Hemisphere. A majority of the improvements persist in the presence of other climate feedbacks in the fully coupled experiments. In addition, warming of the subsurface water over the majority of
\end{abstract}

\footnotetext{
* Corresponding author.

Email address: qing_li_1@brown.edu (Qing Li)
}

Preprint submitted to Ocean Modeling

July 24, 2015 
global ocean is observed in the fully coupled experiments with waves, and the cold subsurface ocean temperature biases are reduced.

Keywords: Langmuir mixing, Mixed layer, KPP, Climate model

1

\section{Introduction}

The climate system is a multi-scale system. Processes at different spatial scales frequently interact with each other, especially in the ocean surface boundary layer. Biases in the mixed layer depth (MLD) of the ocean surface boundary layer lead to biases in dynamically important quantities such as temperature and salinity, as well as in the transport of passive tracers such as the chlorofluorocarbons (CFCs) and nutrients and other biogeochemical tracers (Wang et al. 2012, Long et al. 2013, Moore et al. 2013). MLD biases of both signs are commonly observed in the state-of-the-art climate models (Fox-Kemper et al. 2011 Danabasoglu et al. 2012; Belcher et al. 2012), particularly a persistent shallow bias in distinct regions of the Southern Ocean (Sallée et al., 2013). Accurate modeling of the climate therefore requires better representation of processes in the ocean surface boundary layer from large to small scales.

A recent parameterization of submesoscale eddies in the ocean mixed layer substantially improves the simulation of the upper ocean climate (Fox-Kemper et al. 2008, 2011). While the restratification of ocean surface layer by the submesoscale eddies reduces or eliminates many deep MLD biases, it exacerbates the shallow MLD bias in the Southern Ocean. Hence, even smaller scale phenomena, such as ocean surface gravity waves, are believed to contribute to this longstanding model bias (Belcher et al. 2012). Langmuir mixing in particular is one potential mechanism that typically deepens the MLD, even in the presence of mixed layer eddy restratification nearby (Hamlington et al., 2014). Since Langmuir mixing and submesoscale eddy restratification respond to very different forcings, the deepening and shoaling effects do not necessarily cancel out. Instead the deepening and shoaling induced by the two mechanisms will occur separately depending on the ocean state and forcings. This study there- 
fore attempts to assess the impact of surface gravity waves on the MLD and global climate by parameterizing Langmuir mixing in a climate model that also uses a submesoscale eddy restratification parameterization (Fox-Kemper et al. 2008, 2011).

It is well know that ocean surface gravity waves can influence the climate through a variety of processes at the ocean-atmosphere interface Cavaleri et al. 2012). However, few of them are explicitly represented in the state-of-the-art climate models at present. Efforts to model the bulk wave influences have focused mainly on two processes: (a) wave breaking (Sullivan et al., 2004) and (b) Langmuir circulation (Langmuir, 1938) and its turbulent form, Langmuir turbulence (McWilliams et al., 1997, Sullivan and McWilliams, 2010). While the mixing and dissipation induced by wave breaking decay rapidly away from the surface, Langmuir turbulence can elevate the turbulent kinetic energy and mixing throughout the mixed layer and is therefore believed to be a major mechanism in deepening the mixed layer (Kantha and Clayson, 2004, D'Asaro et al. 2014). Langmuir turbulence has been studied extensively with Large Eddy Simulations (LES) of the wave-averaged or Craik-Leibovich equations (e.g. McWilliams et al., 1997; McWilliams and Sullivan, 2000; Harcourt and D'Asaro, 2008; Van Roekel et al. 2012; Hamlington et al., 2014). A diagnostic study by Belcher et al. (2012) suggests that turbulent energy available for mixing in the ocean surface boundary layer is underestimated without forcing by the surface waves, which is verified by Sutherland et al. (2014a) with summer observations in the North Atlantic. Using a second moment closure model (Harcourt, 2013), D'Asaro et al. (2014) found roughly a factor of two greater turbulent kinetic energy within the mixed layer, and deepening of MLD by $15-20 \%$ on average at high latitudes when Langmuir turbulence was included.

Efforts have been made to estimate the bulk effects of Langmuir mixing by scaling the vertical turbulent kinetic energy (VKE) with parameters such as

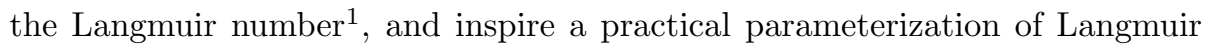

\footnotetext{
${ }^{1} \mathrm{~A}$ bulk measure of the relative importance of shear and Stokes forcing, with smaller values
} 
mixing by modifying the K-profile parameterization (KPP; Large et al., 1994). McWilliams and Sullivan (2000, hereafter MS2K) first attempted to account for Langmuir mixing in KPP by introducing an enhancement factor to the turbulent velocity scale based on the turbulent Langmuir number $L a_{t}$ (McWilliams et al. 1997). Smyth et al. (2002, hereafter SS02) refined the MS2K scheme by adding a modulation to the enhancement factor by stable or convective stratification conditions. However, both of these schemes assume simplistic relationships between wind and waves, e.g., assuming wind and waves are aligned, which are uncommon in the real world (Hanley et al., 2010; Webb and Fox-Kemper, 2015). In addition, the Stokes drift magnitudes integrated over the wavenumber-direction wave spectrum typically decay faster than exponentially with depth (Webb and Fox-Kemper, 2011). Since $L a_{t}$ only uses surface magnitudes, it may not correctly estimate the relative effect of Stokes forcing to shear forcing. To account for the penetration depth of Stokes drift versus the surface layer depth, Harcourt and D'Asaro (2008) proposed a VKE scaling predicted by a surface layer averaged Langmuir number, $L a_{S L}$. To account for the effect of misaligned wind and waves, Van Roekel et al. (2012, hereafter VR12) proposed a new VKE scaling predicted by the surface layer averaged and projected Langmuir number, $L a_{S L, p r o j}$. The basic idea is that when the wind and waves are misaligned, both the friction and Stokes drift velocities must be projected into the Langmuir cell direction, which lies in between, to represent the important portion of the wind induced shear and Stokes forcing. It generally reduces the influence of Langmuir turbulence when misaligned wind and waves occur.

These scalings were tuned to best match the statistics of LES, but rarely have they been tested in a climate model. To our knowledge, only one study

indicating stronger wave effects. It has various definitions to account for different aspects of the wind wave relation. The simplest one is the turbulent Langmuir number, $L a_{t}$, first introduced by McWilliams et al. (1997) and defined in (5). Other definitions include the surface layer averaged Langmuir number, $L a_{S L}$, introduced by Harcourt and D'Asaro (2008) and defined in their (27), and the surface layer averaged and projected Langmuir number, $L a_{S L, \text { proj }}$, introduced by Van Roekel et al. (2012) and defined in 11. 
(Fan and Griffies, 2014) has implemented and tested MS2K and SS02 scalings in a climate model, the Geophysical Fluid Dynamics Laboratory (GFDL) Climate Model (CM2M). SS02 is their preferred scheme, improving the MLD and sea surface temperature (SST) most. However, as will be shown later, both schemes introduce too much mixing when implemented in the National Center for Atmospheric Research (NCAR) Community Earth System Model, version 1.2 (CESM1.2). This is one of our motivations to use the newer scalings, as well as a desire to quantify the novel physical processes. It should be noted that the phenomenology and behavior of Langmuir turbulence are better described in LES work (See, e.g., McWilliams et al., 1997, Harcourt and D'Asaro, 2008, Grant and Belcher, 2009, Van Roekel et al. 2012). Our primary goal is to test the newly proposed VR12 scaling with $L a_{S L, p r o j}$ in CESM, thereby assessing the impact of Langmuir mixing on global climate. This is the first time this scaling has been tested in a state-of-the-art climate model.

A third generation wave model WAVEWATCH III is coupled with CESM1.2 to provide the wave spectrum for calculating the Stokes drift velocity and Langmuir number. Here, Langmuir mixing is parameterized with different VKE scalings in KPP and its impacts on the MLD, ocean ventilation, as well as ocean temperature and salinity, are assessed in this coupled climate model. It should be noted that this work represents a first step towards more comprehensive studies on the influences of ocean surface gravity waves on the global climate. In this study the effects of Langmuir mixing are parameterized in the climate model through KPP in the most straightforward way. Wave breaking is not considered here, nor the wave influences on the atmosphere from roughening the ocean surface beyond the ensemble average effects related to wind speed. The set of parameterizations explored here for Langmuir mixing in KPP could easily be implemented in any other climate models using KPP and a coupled wave model to provide the Stokes drift velocity.

The details of coupling WAVEWATCH III in CESM1.2 and parameterizing Langmuir mixing in KPP are described in Section 2, followed by their impact on MLD, water mass ventilation, and ocean temperature and salinity in Section 
3. We will summarize in Section 4 and close with a brief discussion in Section 5 .

\section{Methods}

\subsection{Coupling WAVEWATCH III in CESM1.2}

A modified version of WAVEWATCH III (version 3.14; Tolman, 2009), an operational third generation wave model developed by the National Oceanic and Atmospheric Administration (NOAA) and the National Centers for Environmental Prediction (NCEP), has been incorporated into the NCAR Community Earth System Model, version 1.2 framework (CESM1.2; Gent et al., 2011 Vertenstein et al. 2013) as a new active model component. All model physics and settings in this component are based on the work by Rascle et al. (2008) and Ardhuin et al. (2010)

A schematic diagram of WAVEWATCH III coupling in CESM1.2 is displayed in Fig. 1. As illustrated, WAVEWATCH III is now two-way coupled with the ocean component, the Parallel Ocean Program version 2 (POP2; Smith et al. 2010), through the central coupler (CPL7; Craig et al., 2011), but only one-way coupled with the atmosphere and sea ice components. WAVEWATCH III generates and evolves the wave action density spectrum in a coupled spatial-spectral domain; it is forced by sea surface temperatures (SSTs) and currents from the ocean model, and by surface air temperatures, $10 \mathrm{~m}$ wind velocities, and ice fraction concentrations, from either an active atmosphere and sea ice model or from forcing datasets such as the inter-annually varying Coordinated Ocean-ice Reference Experiments phase II atmospheric data sets (CORE-II; Large and Yeager, 2009). WAVEWATCH III passes back to the ocean model the surface Stokes drift (see next paragraph), Langmuir number (various definitions following McWilliams et al., 1997, Harcourt and D'Asaro, 2008; Van Roekel et al.

\footnotetext{
${ }^{2}$ The relevant compile switches are 'ST3', 'STAB3', and 'FLX0'; all others are default. Further discussion on the choice of source terms will be delayed until Section 5
} 
2012 ) and the angles between wind and Langmuir cells (as part of the enhancement factor following Van Roekel et al. 2012, see Section 2.2.3 for detail). The boundary layer depth diagnosed in the ocean model is also an input variable for WAVEWATCH III to calculate the surface layer averaged Langmuir number, $L a_{S L}$, and the angle between wind and Langmuir cells.

It should be noted that two forms of Stokes drift are used for calculating the Langmuir number and are diagnosed within WAVEWATCH III using the frequency-direction spectrum and a parametric $f^{-5}$ tail assumption (Webb and Fox-Kemper, 2011; Webb, 2013). It is necessary to pass the first, the surface Stokes drift, to the ocean model in order to estimate the wave induced entrainment from below the ocean surface boundary layer (see Section 2.2.4 for more detail). The second, a surface layer averaged Stokes drift, relies on the mixed layer depth from the ocean model and is used when the effects of misaligned wind and waves and Stokes drift penetration depth are considered (see (11)). Since this latter form has been depth-integrated analytically, there is no concern that the faster than exponential decay in Stokes drift is not resolved by the coarse vertical resolution of the climate ocean model grid.

The coupling frequency of WAVEWATCH III with other model components is the same as that for the atmosphere and sea ice components - the waveatmosphere coupling interval is 30 minutes if running active atmosphere and sea ice models, and 6 hours if using CORE datasets. Since the ocean model communicates with the coupler once per day, the wave-ocean coupling is not as frequent as the wave-atmosphere coupling. However, the short-time misalignment of wind and waves may be captured here since it depends on the waveatmosphere coupling frequency, not the wave-ocean coupling frequency. Further work will assess the importance of the diurnal cycle of wave-forced turbulence versus other high-frequency ocean coupling effects, such as near-inertial shear driven mixing (Jochum et al. 2013); for now the coupling frequency to POP2 is too low in the base CESM1.2 configuration to examine such phenomena.

Running WAVEWATCH III over the global ocean with high resolution (e.g. $1^{\circ}$ spatial resolution) is computationally expensive. To capture the wave clima- 
tology well while reducing the cost as much as possible, we need to choose an optimal spatial and spectral resolution for WAVEWATCH III before incorporating it into CESM. Four different grids, G1F25T24, G1F13T12, G2F25T24 and G3F25T24, have been tested in this study, which are summarized in Table 1. We have tested each grid by running WAVEWATCH III alone with COREII interannual forcing data for an arbitrarily chosen month, here the month of August, 2000. Surface Stokes drift magnitudes for three coarser resolutions are compared with a higher control resolution, G1F25T24, and root mean square errors (RMSEs) and the relative errors are tabulated in Table 1. Coarsening the spectral grid resolution increases the error more so than coarsening the spatial grid resolution. In addition, the computational cost is not significantly reduced on the coarser spectral grid; in contrast, WAVEWATCH III performs much faster on the coarser spatial grid both in CPU hours and wall clock measures (Webb, 2013). G3F25T24 therefore appears to be the best choice of those considered, with a relative error of $11.2 \%$ for surface Stokes drift (corresponding to a relative error in the enhancement factor of no greater than $16.8 \%$ ) versus the much more expensive G1F25T24 model. It should be noted that polar ice caps were used in this grid, and as such, we are assuming that there are no wave effects north of $\sim 78^{\circ} \mathrm{N}$ in the Arctic 3 While future longer simulations would need to address this shortcoming, it is not expected to significantly affect lower latitude Stokes drift velocities, which are the dynamical emphasis of this study.

\subsection{Parameterizing Langmuir mixing in KPP}

Parameterizing Langmuir mixing in the K-profile parameterization (KPP; Large et al., 1994) involves two processes to account for the effects of Langmuir turbulence: first, enhanced vertical turbulent kinetic energy (VKE) within the ocean surface boundary layer and second, deeper boundary layer penetration.

\footnotetext{
${ }^{3}$ The ice lines within WAVEWATCH III were chosen to maximize the latitudinal regions covered without degrading model performance by using smaller time steps to account for shrinking grid cell widths.
} 
The former is parameterized by an enhancement factor applied to the turbulent velocity scale, for which different scalings have been proposed. The second accounts for eddies formed near the surface, or within a Langmuir cell being more energetic due to the additional Stokes, or Langmuir velocity, so that they are able to penetrate deeper into the stratification below. Five different experiments are designed to explore the impact of different aspects of Langmuir mixing on global climate through these effects, which are described below and summarized in Table 2 for quick reference.

\subsubsection{Mc Williams and Sullivan (2000) parameterization of VKE}

Following the work of McWilliams and Sullivan (2000), we introduce an enhancement factor $\mathcal{E}$ to the turbulent velocity scale in KPP

$$
W=\frac{k u^{*}}{\phi} \mathcal{E}
$$

with $k=0.4$ the von Kármán constant, $u^{*} \equiv \sqrt{|\boldsymbol{\tau}| / \rho_{0}}$ the magnitude of surface friction velocity ( $\boldsymbol{\tau}$ is the surface wind stress and $\rho_{0}$ is the surface ocean density) and $\phi$ the dimensionless flux profile.

The enhanced turbulent velocity scale affects the vertical viscosity, tracer diffusivity and nonlocal flux in KPP. In particular, the eddy diffusivity profile

$$
\kappa_{v}=W h G(\sigma)
$$

with $h$ the boundary layer depth, $G(\sigma)$ the shape function, and $\sigma=-z / h$ the normalized depth, is directly amplified. The boundary layer depth is determined as the smallest depth at which the bulk Richardson number reaches a critical value,

$$
R i_{b}(h)=\frac{h\left[b_{r}-b(h)\right]}{\left|\boldsymbol{u}_{r}-\boldsymbol{u}(h)\right|^{2}+U_{t}^{2}}=R i_{c r},
$$

with $b_{r}$ the surface layer averaged buoyancy and $\boldsymbol{u}_{r}$ the surface layer averaged velocity. The critical bulk Richardson number $R i_{c r}=0.3$ is empirically determined from observations. Therefore, the boundary layer depth is deepened as a result of the enhanced turbulent velocity scale through the parameterized 
unresolved vertical shear $U_{t}^{2}$ (which is proportional to $W$, Equation 23 in Large et al. (1994)) in the bulk Richardson number.

McWilliams and Sullivan (2000) propose the enhancement factor as

$$
\mathcal{E}=\sqrt{1+0.08 L a_{t}^{-4}} \approx \sqrt{1+\left(1.88 L a_{t}\right)^{-4}}
$$

to best fit their LES results.

$$
L a_{t}=\sqrt{\frac{u^{*}}{\left|\boldsymbol{u}_{s}(0)\right|}}
$$

is the turbulent Langmuir number (McWilliams et al., 1997), with $\boldsymbol{u}_{s}(0)$ the surface value of Stokes drift velocity. Hereafter we will refer to the parameterization scheme described by (3), (4), and (5) as MS2K.

\subsubsection{Smyth et al. (2002) parameterization of VKE}

Instead of using a constant coefficient in the enhancement factor (4), Smyth et al. (2002) propose a modified version

$$
\mathcal{E}=\sqrt{1+\mathcal{C}_{w}\left(u^{*}, w^{*}\right) L a_{t}^{-4}}
$$

in which

$$
\mathcal{C}_{w}\left(u^{*}, w^{*}\right)=0.15\left(\frac{u^{* 3}}{u^{* 3}+0.6 w^{* 3}}\right)^{2} \approx\left(1.61\left(\frac{u^{* 3}+0.6 w^{* 3}}{u^{* 3}}\right)^{1 / 2}\right)^{-4}
$$

3ere, $w^{*}$ is the convective velocity scale defined as

$$
w^{*}=\left(-B_{f} h\right)^{1 / 3},
$$

and $B_{f}$ is the surface buoyancy flux. This modification enhances the effect of 32 Langmuir turbulence in a stable (wind-forced, positive surface buoyancy flux) condition and reduces it in a convective (negative surface buoyancy flux) con34 dition. Heuristically, this is similar to the effect achieved by the Harcourt and 235 D'Asaro (2008) $L a_{S L}$ below. Hereafter, we refer to this parameterization described by (6) and (7) together with (3) and (5) as SS02. 


\subsubsection{Van Roekel et al. (2012) parameterization of VKE}

In both MS2K and SS02, the relative importance of wind induced shear and Stokes forcing are represented by $L a_{t}$. Both studies assume that Stokes drift is aligned with the surface wind stress, which is not always the case. In fact, the ocean is rarely in wind-wave equilibrium (Hanley et al. 2010). Van Roekel et al. (2012) propose a projected Langmuir number,

$$
L a_{\text {proj }}=\sqrt{\frac{u^{*} \cos (\alpha)}{\left|\boldsymbol{u}_{s}(0)\right| \cos \left(\theta_{w w}-\alpha\right)}},
$$

to account for the influence by the misalignment of wind and waves. Here $\alpha$ is the angle between wind and Langmuir cells, and $\theta_{w w}$ is the angle between ${ }_{\square}$ Stokes drift and wind. Note that wave orientation depends on depth Webb and Fox-Kemper, 2015), but here only one wave direction is calculated, which tends to be more aligned with wind for the $L a_{t}$ definition (9) than the $L a_{S L}$ definition (as defined in (11) below), as surface Stokes drift is more aligned with wind than depth-integrated Stokes drift (Webb and Fox-Kemper, 2015).

Van Roekel et al. (2012) also suggest that a suitable estimation of $\alpha$ could be made by

$$
\alpha_{L O W} \approx \tan ^{-1}\left[\frac{\sin \left(\theta_{w w}\right)}{\frac{u^{*}}{u_{s}(0) k} \ln \left(\left|H_{M L} / z_{1}\right|\right)+\cos \left(\theta_{w w}\right)}\right],
$$

in which $H_{M L}$ represents a relevant depth scale, as discussed below, and $z_{1}$ is the onset depth of the law of the wall, which could be taken as four times the significant wave height (Thorpe, 2007). There are at least two ways of conceptualizing the relevant depth scale $H_{M L}$. The first is to estimate the mixing depth to which active turbulence is expected under the present forcing (as the KPP boundary layer depth). The second is to evaluate a mixed layer depth, based on the present stratification of active tracers and thus the historical depths of turbulent mixing. As noted by Van Roekel et al. (2012), it might be more realistic to take $H_{M L}$ as the mixing depth (the KPP boundary layer depth as implemented in our simulations). In our model, the mixed layer depth is consistently deeper than the mixing depth by as much as $20 \mathrm{~m}$ in summer 
and up to $200 \mathrm{~m}$ differences in winter convection regions. So using mixing depth here gives a higher estimate of $\alpha$. However, the relation between the mixing layer depth and the mixed layer depth could be complicated in other models and observations (e.g. Noh and Lee, 2008, Sutherland et al., 2014b). When the Langmuir cells are aligned with wind (i.e., $\alpha \rightarrow 0$ ), $L a_{\text {proj }}$ reduces to $L a_{t}$.

Combined with the influence of Stokes drift penetration depth (Harcourt and D'Asaro, 2008), Van Roekel et al. (2012) propose another Langmuir number, the surface layer averaged and projected Langmuir number,

$$
L a_{S L, p r o j}=\sqrt{\frac{u^{*} \cos (\alpha)}{\left|\left\langle\boldsymbol{u}_{s}\right\rangle_{S L}\right| \cos \left(\theta_{w w}-\alpha\right)}} .
$$

Here \langle\rangle$_{S L}$ denotes an average over the surface layer, which is defined as the upper $20 \%$ of the mixed layer ${ }^{4}$ by Harcourt and D'Asaro (2008), as confirmed by Van Roekel et al. (2012).

The VKE scaling by Van Roekel et al. (2012) suggests a different formula for the enhancement factor,

$$
\mathcal{E}=|\cos \alpha| \sqrt{1+\left(c_{1} L a_{x}\right)^{-2}+\left(c_{2} L a_{x}\right)^{-4}}
$$

in which $L a_{x}$ represents either $L a_{t}, L a_{\text {proj }}$ or $L a_{S L, p r o j}$. The value of constants $c_{1}$ and $c_{2}$ depends on the choice of Langmuir number. Van Roekel et al. (2012) proposed that for $L a_{\text {proj }}, c_{1}=3.1, c_{2}=5.4$ and for $L a_{S L, p r o j}, c_{1}=1.5, c_{2}=5.4$. Reiterate that all parameterization scaling factors are taken from LES results, and are not adapted based on the outcomes in the coarse resolution general circulation models (GCMs). Thus, no additional tuning occurs to optimize the GCM results, and indeed tuning the coefficients based on GCM results would be likely to confuse different model biases, such as Langmuir mixing versus wave breaking and near-inertial mixing.

The parameterization described by $(10,11)$, and $(12)$, with $L a_{x}=L a_{S L, p r o j}$, will be referred to as VR12-MA. As a comparison to assess the effects of mis-

\footnotetext{
${ }^{4}$ Again, we are using the KPP boundary layer instead of the mixed layer in our experiments to be consistent with the choice in $\alpha$.
} 
aligned wind and waves and Stokes drift penetration depth, an aligned case, VR12-AL, is also tested. It assumes $\alpha=0$ in the enhancement factor formula in 12 with $L a_{x}=L a_{\text {proj }}$, which reduces to $L a_{t}$. In this case the only difference from MS2K is the enhancement factor scaling.

\subsubsection{Parameterization of boundary layer depth and entrainment}

The boundary layer depth, $h$, determined from (3), represents the maximum depth that turbulent eddies formed in the boundary layer can penetrate into the stratification given by buoyancy profile, $b(z)$. It is assumed these most deeply penetrating eddies form in the surface layer, which gives them the average buoyancy, $b_{r}$, and velocity, $\boldsymbol{u}_{r}$, of the layer. For present purposes the Langmuir and Stokes velocities are both assumed to scale with $\boldsymbol{u}_{s}(0)$, but are not distinguished, and their effects are represented by including the square of surface Stokes drift $\left|\boldsymbol{u}_{s}(0)\right|^{2}$ in the denominator of the bulk Richardson number as

$$
R i_{b}(z)=\frac{z\left[b_{r}-b(z)\right]}{\left|\boldsymbol{u}_{r}-\boldsymbol{u}(z)\right|^{2}+U_{t}^{2}+\left|\boldsymbol{u}_{s}(0)\right|^{2}}
$$

The boundary layer depth is then determined by matching 13 to the critical Richardson number, $R i_{c r}$, as in (3). This parameterization of boundary layer entrainment with the Van Roekel et al. (2012) VKE scaling will be referred to as VR12-EN.

Note that this might overestimate the influence of Stokes shear due to the "anti-Stokes" effect, i.e., in some cases, part of the Eulerian and the Stokes velocity will in fact cancel, leaving a residual velocity whose vertical shear impacts the bulk Richardson number (McWilliams and Fox-Kemper, 2013; Haney et al. 2014). Another source of overestimation is the use of surface Stokes drift instead of a surface layer averaged value, as used for $b_{r}$ and $\boldsymbol{u}_{r}$. In addition, wave effects were present in the observations used to determine $R i_{c r}=0.3$ in (3); therefore using the same value in 13 would also tend to overestimate $h$. However, a compensating factor is that perhaps the surface Stokes drift velocity should be added to $\boldsymbol{u}_{r}$ in 13 and then sum squared. 
As mentioned before, this set of empirical parameterizations, to include Langmuir mixing in a climate model, is intended as a starting point toward more comprehensive parameterizations (Harcourt, 2013, Grant and Belcher, per. comm.). With these choices, it is easy to turn on the effects of misaligned wind and waves and Stokes drift penetration depth on amplifying VKE, and the effects of Stokes shear on the boundary layer depth in steps to estimate their influences individually. Note that some recent studies, especially McWilliams et al. (2014), propose more sophisticated scalings for entrainment rate. Exploring these alternatives will be left to future work.

\subsection{Experimental setup}

Two sets of experiments are conducted in this study: 1) Wave-Ocean only experiments, with all the parameterization schemes described in the previous section, and 2) fully coupled experiments for CTRL and VR12-MA.

In all the simulations presented here, the wave model WAVEWATCH III is integrated on the G3F25T24 grid, described in Table 1. The ocean model POP2 is integrated on a nominal $3^{\circ}$ horizontal grid with 60 vertical levels (referred to as gx3v7 in the CESM terminology). The horizontal resolution is enhanced in the Tropics and high latitudes. The vertical grid spacing is $10 \mathrm{~m}$ in the upper $160 \mathrm{~m}$, increasing to $250 \mathrm{~m}$ by the depth of around $3500 \mathrm{~m}$, then remaining constant to the bottom at $5500 \mathrm{~m}$.

All of the Wave-Ocean experiments have been integrated for four CORE-II interannual forcing cycles (248 years) $)^{5}$. Unless otherwise noted, we define the mean states as the 48-year time-means for model years 200-247, corresponding to year 1961-2008, avoiding the large adjustments associated with the unphysical jump in the forcing from 2009 back to 1948 (Danabasoglu et al., 2014).

In the fully coupled experiments, the Wave-Ocean model is coupled to an

\footnotetext{
${ }^{5}$ One cycle of CORE-II forcing represents a 62-year period, corresponding to 1948-2009. Note that the CORE-II protocol calls for five forcing cycles. For our purpose here four forcing cycles are adequate.
} 
atmosphere model (CAM4) and a land model (CLM4) running on the T31 grid, and a sea ice model (CICE4) running on the same nominal 3-degree grid as the ocean model, with a wave-atmosphere-ice-land coupling interval of half an hour. The wave-ocean coupling frequency remains once per day. Preliminary fully coupled experiments are integrated for 70 years. The average of the last 20 years are analyzed in this study.

\section{Results}

\subsection{Vertical turbulent kinetic energy}

To illustrate the impact of misaligned wind and waves and the penetration depth of Stokes drift on scaling the bulk effects of Langmuir mixing, Fig. 2 shows the mixed layer mean VKE normalized by the friction velocity squared, calculated from the scalings with $L a_{t}, L a_{\text {proj }}$ (to account for misaligned wind and waves), $L a_{S L}$ (to account for the effect of Stokes drift penetration depth), and $L a_{S L, p r o j}$ (to account for both), following Van Roekel et al. (2012), for arbitrarily chosen winter and summer months (Jan. and Jul. of model year 247). In the no wave limit $(L a \rightarrow \infty)$, the normalized VKE is 0.6 , determined using simulations that only include shear turbulence (see more in Van Roekel et al. 2012). It is also important to note that misaligned wind and waves could potentially reduce the normalized VKE in comparison with the no wave case (leading to a normalized VKE less than 0.6) when the angles between wind and waves, and therefore the angles between wind and Langmuir cells, are large (due to the $\cos \alpha$ term in $(12)$ ). However, this is rarely seen here presumably as a result of the relatively coarse spatial resolution used in the ocean and wave models, which limits refraction by coastal and current features.

The effects of misaligned wind and waves and the penetration depth of Stokes drift could be seen, respectively, by comparing the $L a_{p r o j}$ case and $L a_{S L}$ case with the $L a_{t}$ case. Generally, VKE scaled by $L a_{\text {proj }}$ is smaller than that scaled by $L a_{t}$ almost everywhere (Fig. 2 b,f versus 2 a,e), suggesting that misalignment of wind and waves prevails in the open ocean, and that scaling by $L a_{t}$ assum- 
ing alignment overestimates the Langmuir mixing. Scaling by $L a_{S L}$ (Fig. 2, g versus 2 a,e) show the enhanced VKE is more vigorous in the summer hemispheres where the mixed layer depth (MLD) is shallow and less vigorous in the winter hemispheres where the MLD is deep. This is because the VKE is sensitive to the ratio of the penetration depth of Stokes drift to the MLD which varies seasonally. The VKE increases monotonically with this ratio, with small values occurring for deep MLD (Harcourt and D'Asaro, 2008). These two effects are partly canceled in the summer hemisphere but reinforced in the winter hemisphere. Therefore, the amount of enhancement in VKE with or without the influences by the wind-wave misalignment and the Stokes drift penetration depth are similar in the summer hemisphere but very different in the winter hemisphere, with much smaller VKE enhancement observed in the $L a_{S L, p r o j}$ scaling. As in Fan and Griffies (2014), it will be shown that overmixing in the winter hemisphere can be problematic.

\subsection{Mixed layer depth}

The direct effect of including Langmuir mixing in KPP is the deepening of the MLD through enhanced surface ocean mixing. In order to directly compare the model results with observations, we calculate the MLD following the density threshold method in de Boyer Montégut et al. (2004) as the depth where the potential density (referenced to surface) changes by $0.03 \mathrm{~kg} \mathrm{~m}^{-3}$ from its surface value, rather than using the default MLD definition implemented in POP2 with the buoyancy gradient criterion (Large et al. 1997) both definitions are tested and the qualitative effects of Langmuir mixing shown here do not depend on the diagnostic definition of MLD. The main reason to choose the density criterion definition is to have a matched observation for comparison. Different definitions for MLD agree in the extratropical regions 7

\footnotetext{
${ }^{6}$ Note that for both definitions MLD is calculated online at each time step, then averaged to output the monthly mean.

${ }^{7}$ For clarity regions poleward of $30^{\circ} \mathrm{N} / \mathrm{S}$ are referred to as extratropical regions, while regions between $30^{\circ} \mathrm{S}$ and $30^{\circ} \mathrm{N}$ are referred to as equatorial regions.
} 
but MLD in the equatorial regions varies among definitions.

Fig. 3 and 4 show the summer and winter mean MLD, respectively, from different experiments versus the MLD observed climatology de Boyer Montégut et al. 2004) with the same density criterion, updated to include the ARGO data to 2012. The subfigures $3 \mathrm{~b}$ and $4 \mathrm{~b}$ summarize the latitudinal distribution of root mean square errors (RMSEs) for each experiment. In addition, the percentage change of the summer and winter mean MLDs in VR12-MA and VR12-EN versus CTRL are shown in Fig. 5 to highlight the net effects of Langmuir mixing on MLD.

A shallow bias of summer mean MLD in the Southern Ocean is clearly seen in CTRL (Fig. 3a,c and black line in 3b) with no Langmuir mixing effect included. By simply implementing an enhancement factor to the turbulent velocity scale in KPP scaled by $L a_{t}$ as in (4), MS2K generally introduces too much mixing, greatly increasing the RMSE. While the enhanced mixing alleviates the shallow biases in the Southern Ocean, it also introduces a modest deep bias in the equatorial regions (Fig. $3 \mathrm{~d}$ and solid gray line in $3 \mathrm{~b}$ ). Accounting for the modulation to the enhancement factor by stratification conditions, i.e., to magnify the enhancement in stable conditions and restrain it in convective conditions, SS02 seems to correct the over-deepening of MLD in the equatorial regions in MS2K, but it causes even more overmixing than $\mathrm{MS} 2 \mathrm{~K}$ in the extratropical regions (Fig. 3e and dashed gray line in 3p). In the sense of RMSEs (Table 3), MS2K improves the summer MLD simulation in the Southern Ocean but worsens it elsewhere. SS02 generally increases the RMSE of summer MLD everywhere.

Van Roekel et al. (2012) considered the effects of both the misaligned wind and waves and the penetration depth of Stokes depth (Harcourt and D'Asaro, 2008 ) in their recently proposed scaling of VKE with $L a_{S L, p r o j}$. Following the idea of applying an enhancement factor to the turbulent velocity scale in KPP, one interesting experiment could be just to use the new VKE scaling, assuming aligned wind and waves (VR12-AL). In VR12-AL, $L a_{\text {proj }}$ reduces to the $L a_{t}$, but the enhancement factor differs from MS2K. As shown in Fig. 3f and the blue line in $3 \mathrm{~b}$, the shallow biases of MLD in the Southern Ocean are significantly 
reduced by $22 \%$ relative to CTRL (Table 3 . The over-deepened MLD in the equatorial regions observed in MS2K is also improved in this case. Over the global ocean the RMSE in MLD is reduced by about $15 \%$ in comparison with CTRL.

Further accounting for the effects of both the misaligned wind and waves and the penetration depth of Stokes drift (VR12-MA), as shown in Fig. 3p, we get similar results as that in VR12-AL, which is not surprising given the similar Summer Hemisphere VKEs in these two experiments (Fig. 2a,e and $2 \mathrm{~d}, \mathrm{~h}$, see the Southern Hemisphere in Jan. and the Northern Hemisphere in Jul.). There are, however, notable improvements in RMSEs over VR12-MA as expected since more physical realism is included. The MLD bias reductions in VR12-MA relative to CTRL are $27 \%$ in the Southern Ocean and $18 \%$ globally (Table 3). This change has the same spirit as the entrainment scaling proposed by Li and Garrett (1997), although differs in detail. Uncertainties in RMSEs are also given in Table 3 for CTRL and VR12-MA, by calculating the RMSEs of 1000 bootstrap estimates of the 48-year mean MLD $^{8}$. The fact that the $90 \%$ confidence intervals of the summer MLD RMSEs for CTRL and VR12-MA do not overlap indicates that the reductions in summer MLD bias in VR12-MA are significant versus the uncertainty of the mean state estimated by the 48-year time-mean.

Though the exact physics by which Langmuir mixing enhances entrainment is not yet clear, one way to estimate how large this effect could be is to treat Stokes shear as an additional source of the unresolved vertical shear in the definition of bulk Richardson number (VR12-EN; (13)). The resulting summer MLD is shown in Fig. 3h. This is rather an estimation of the upper limit neglecting the "anti-Stokes" Eulerian flow that opposes the Stokes drift (McWilliams

${ }^{8}$ For each bootstrap estimate, 48 years of monthly mean MLD are used. Bootstrapping occurs by choosing 48 versions of each month with replacement, followed by averaging to give an estimate of the mean annual cycle. 1000 versions of this cycle were used to generate the confidence interval. 
and Fox-Kemper, 2013). It should be noted that the Langmuir mixing induced deepening of extratropical MLD, especially in the Southern Ocean, is significant in comparison with the natural variability of MLD, as shown by the dot shading in Fig. 5. Equatorial deepening is statistically significant in VR12-EN, but not VR12-MA or VR12-AL.

Compared with the observations, substantial biases for the winter mean MLD in CTRL occur in the extratropical regions in both hemispheres (Fig. 4 b). There are shallow biases in the Gulf Stream and Kuroshio Extension regions, the Labrador Sea, and the Pacific and Atlantic sectors of the Southern Ocean (Fig. 4a,c and black line in 4b). Similar to the summer MLDs, the winter MLDs get too deep in MS2K and SS02, especially in the extratropical regions. The improvements brought by SS02, compared with MS2K, is that SS02 handles the equatorial regions better. Though the reductions of winter MLD biases in the three versions of the Van Roekel et al. (2012) parameterization are not as substantial as that for the summer MLD, we do get smaller RMSEs of winter MLD in both the global ocean and the Southern Ocean, and these reductions are significant versus the estimated uncertainty of the mean state (Table 3). These improvements mainly come from the deepening winter MLD in the Pacific and Atlantic sectors of the Southern Ocean and the North Pacific, though considerable biases still remain in the North Atlantic, especially the Labrador Sea. However, unlike MS2K and SS02, the Van Roekel et al. (2012) parameterizations do not come at a cost of introducing new statistically significant biases.

One interesting result is the similarity in winter mean MLD between VR12AL and VR12-MA, given the large differences in the winter hemisphere VKEs (Fig. 2 a,e and 2 $\mathrm{d}, \mathrm{h}$, see the Southern Hemisphere in Jul. and the Northern Hemisphere in Jan.). Moreover, the differences between VR12-EN and VR12MA are much greater than those between VR12-MA and VR12-AL. This might be because, as winter MLD is deep, it may be more sensitive to the enhanced entrainment, which directly deepens the ocean boundary layer, rather than enhanced mixing within the already mixed layer. This suggests that improving 
the representation of entrainment may be critical in reducing the winter MLD biases.

The percentage change of the summer and winter mean MLDs in VR12MA and VR12-EN relative to CTRL is shown in Fig. 5, illustrating the net effects of Langmuir mixing on MLD. By introducing Langmuir mixing in KPP, the MLD is increased globally. Predominantly, the summer mean MLD in the Southern Ocean and winter mean MLD in the North Atlantic and North Pacific increase by $20 \%$ (VR12-MA) to $60 \%$ (VR12-EN). All these changes are significant in terms of the MLD standard deviation 9 in CTRL. The degree of relative increase in MLD induced by Langmuir mixing is surprisingly consistent with other studies that use totally different schemes (e.g. D'Asaro et al., 2014), which gives us confidence in the parameterization of Langmuir mixing in KPP in the climate model. D'Asaro et al. (2014) had less summertime deepening, the reasons for which are beyond the scope of the present work.

\section{3. $p C F C-11$}

The Langmuir enhanced surface boundary layer mixing should directly affect ventilation to deeper waters with indirect effects on air-sea gas exchange and energy transfer. Ventilation can be quantified by the response of passive tracers with known atmospheric sources, such as the chlorofluorocarbons (CFCs). CFCs enter the ocean through air-sea interaction at the ocean surface and are inert in the ocean. Their concentration history in the atmosphere is relatively well known, and they have been widely measured in the global ocean during the Global Ocean Data Analysis Project (GLODAP; Key et al. 2004). Therefore, they are good indicators of how mixing effects in the upper ocean affect deeper water masses, which makes them ideal for assessing integrated model biases. Here we use the CFC-11 partial pressure distribution (pCFC-11), as it largely eliminates the influences of temperature biases in the CFC-11 concentration.

To set up the CFC tracers experiment, CFC concentrations in the atmo-

\footnotetext{
${ }^{9}$ Based on monthly mean MLD data.
} 
sphere were set near the end of the third CORE-II cycle, at model year 170 (corresponding to data year 1931), and ran through the end of the fourth cycle. The annual mean pCFC-11 of model year 233 (corresponding to data year 1994) is compared with the GLODAP pCFC-11 data.

Fig. 6 shows the zonal mean pCFC-11 (patm) in the Southern Hemisphere for the GLODAP data and zonal mean pCFC-11 anomaly from the observation for different experiments. In comparison with the observations, substantially low concentration biases are found in CTRL, reflecting insufficient ventilation and mixing in the upper ocean. When Langmuir mixing is parameterized in KPP, MS2K and SS02 actually introduce too much mixing, so pCFC-11 concentrations are too high (Not shown). The corresponding RMSEs increase significantly as shown in Table 4 As expected, VR12-AL, VR12-MA, and VR12-EN improve the surface ocean mixing, enhancing the ventilation in the upper ocean and reducing the pCFC-11 concentration biases. The low concentration biases of pCFC-11 in the Southern Hemisphere are reduced by $8 \%, 6 \%$, and $19 \%$ in VR12-AL, VR12-MA, and VR12-EN, respectively. Similar improvements are observed globally (Table 4).

Though it appears from RMSE that VR12-EN is better than VR12-AL and VR12-MA, VR12-EN introduces a high concentration bias at the surface, presumably by working away at the distinction between mixed layer and pycnocline waters. Similar but smaller high concentration biases also exist in VR12-AL, presumably during winter when VR12-AL mixes more strongly than VR12MA (Fig. 2). Therefore, VR12-MA appears to be the most skillful in terms of enhancing ocean ventilation and reducing the pCFC-11 biases. Overall the Langmuir effects on ventilation and the bias pattern are small, suggesting that boundary layer mixing is not the dominant source of bias; with possible candidates including the surface forcing, the general circulation, the mesoscale eddy parameterization and neglect of anisotropy in particular (Fox-Kemper et al. 2013 , Reckinger and Fox-Kemper, 2015, Abernathey et al., 2015). 


\section{4. $M L D$ in the fully coupled experiment}

The influences of parameterizing Langmuir mixing in KPP on the MLD described above are further examined in the fully coupled experiments. Fig. 7 illustrates the summer and winter mean MLD averaged over the last 20 years of the 70-year runs without Langmuir mixing (CTRL) and with Langmuir mixing (VR12-MA). Though the wave effect is much smaller than that in the WaveOcean only experiments, the deepening of the summer MLD in the Southern Ocean and winter MLD in the extratropical regions in both hemispheres are clearly seen when Langmuir mixing is included. Similar with analyses in the Wave-Ocean only experiments, we also calculated the RMSEs of summer and winter mean MLD in CTRL and VR12-MA compared with the observations in different regions, as recorded in parentheses in Table 3 . In comparison with CTRL, the RMSEs of summer and winter MLD in VR12-MA are reduced by $17 \%$ and $10 \%$ for the Southern ocean, $12 \%$ and $8 \%$ for the global ocean.

In the fully coupled experiments, the wave-atmosphere-ice coupling frequency is much higher than that in the Wave-Ocean only experiments (once per 30 minutes versus once per 6 hours). In theory, more short timescale processes and more Langmuir mixing effects should be captured. However, a smaller impact of Langmuir mixing on MLD is observed. Note that much of the global wave field results from storminess, which elevates the turbulent kinetic energy and generates waves in the surface ocean. Here the wind forcing is from an active atmosphere model (CAM4) running on the T31 grid, and at T31 CAM4 resolution storminess will be much weaker than in CORE-II. Therefore, smaller responses in the fully coupled experiments are actually expected. More prominent effects of Langmuir mixing should result when the atmosphere model is running on a higher resolution grid (e.g., Kirtman et al., 2011; McClean et al. 2011).

Similar with Fan and Griffies (2014), we also find a strengthening of the Atlantic meridional overturning circulation (AMOC) by about $1 \mathrm{~Sv}(1 \mathrm{~Sv} \equiv$ $\left.10^{6} \mathrm{~m}^{3} \mathrm{~s}^{-1}\right)$ with waves, presumably due to enhanced winter deep convection in the polar North Atlantic (Fig. 7f,d). As a result, the Atlantic meridional heat 
transport is increased by $0.015 \mathrm{PW}\left(1 \mathrm{PW} \equiv 10^{15} \mathrm{~W}\right)$ on average. However, the shallow MLD biases in the Labrador Sea remain in our experiments with Langmuir mixing.

\subsection{Temperature and salinity in the fully coupled experiment}

Enhanced surface ocean mixing by Langmuir turbulence also affects the surface and subsurface ocean temperature. Fig. 8 shows the maps of errors in simulations of annual mean SST and temperature at the depth of $100 \mathrm{~m}$ for CTRL and VR12-MA. The errors are computed as model minus observation, where the observations are from the Polar Science Center Hydrographic Clima„ology (PHC3) dataset. The PHC3 dataset represents a blending of Levitus et al. (1998) data and Steele et al. (2001) data for the Arctic Ocean. In this section, we define the southern extratropical regions, the equatorial regions and the northern extratropical regions as south of $30^{\circ} \mathrm{S}, 30^{\circ} \mathrm{S}$ to $30^{\circ} \mathrm{N}$ and north of $30^{\circ} \mathrm{N}$, respectively.

For SST in CTRL, cold biases are clearly observed in the northern extratropical regions, while biases of both signs are observed in the Southern Ocean. Unlike SST, the subsurface temperature shows cold biases over most part of global ocean except in the northern equatorial Pacific and regions in the Southern Ocean, presumably induced by less than enough ventilation over the global ocean. When Langmuir mixing is included (VR12-MA), SST in the extratropical regions get warmer, reducing cold biases in the Northern Hemisphere (RMSE of $2.90{ }^{\circ} \mathrm{C}$ in VR12-MA versus $3.01{ }^{\circ} \mathrm{C}$ in CTRL; see Table 5 while increasing both the warm and cold SST biases in the Southern Hemisphere (RMSE of 1.04 ${ }^{\circ} \mathrm{C}$ in VR12-MA versus $0.90{ }^{\circ} \mathrm{C}$ in CTRL). The reduction of cold SST biases in the Northern Hemisphere is mostly related to the deepening of winter MLD there, while the increasing SST biases in the Southern Ocean may come from the biases of the position of winter MLD maximum, which is too far poleward than the observation (Fig. 4 and 7f,d). Although the winter MLD is deepened due to the enhance mixing, the errors in the position of winter MLD maximum remain unfixed. Overall, the impact of Langmuir mixing on the global mean 
SST is small, with RMSE of $1.54{ }^{\circ} \mathrm{C}$ in VR12-MA versus $1.53{ }^{\circ} \mathrm{C}$ in CTRL (Table 5). However, enhanced surface ocean mixing increases the subsurface ocean temperature (as illustrated by the temperature at a depth of $100 \mathrm{~m}$ ), reducing the cold biases over the global ocean (RMSE of $1.75^{\circ} \mathrm{C}$ in VR12-MA versus 1.96 ${ }^{\circ} \mathrm{C}$ in CTRL). The RMSEs in the southern extratropical regions, the equatorial regions and the northern extratropical regions are reduced by $7 \%, 17 \%$ and $4 \%$, respectively (Table 5). These improvements come from the warming effects of Langmuir mixing on the subsurface water, presumably through enhanced ocean surface mixing and ventilation discussed above.

To illustrate the impact of Langmuir mixing on the subsurface ocean temperature, Fig. 9 shows the RMSEs of temperature versus depth computed over the global ocean (black), the southern extratropical regions (red), the equatorial regions (blue) and the northern extratropical regions (green) for CTRL (line) and VR12-MA (dash). The monthly mean ocean temperature in both simulations are compared against the PHC3 monthly climatology. Thus, errors in seasonality as well as mean state are detectable in Fig. 9. Note that this is slightly different from that shown in Table 5 and Fig. 8, in which the annual mean ocean temperature is compared against the observation. Overall, the RMSEs of temperature are smaller in VR12-MA than that in CTRL at all depths except near the surface for the southern extratropical regions and the equatorial regions. The most significant improvements appear at the depth of around $100 \mathrm{~m}$. These reductions of RMSE could be divided into reduction of errors in the annual mean temperature and reduction of errors in the seasonal cycle of temperature (not shown). It is found that most of the reductions of RMSE come from the improvement in the annual mean temperature. However, small improvements in the seasonal cycle are also observed near the surface, especially for the southern extratropical regions, though in the southern extratropical regions the RMSE of the annual mean temperature is greater when Langmuir mixing is included.

Unlike the warming effects of Langmuir mixing on the subsurface temperature over the global ocean, Langmuir mixing appears to have little influence 
on the distribution of salinity (not shown). The errors in the simulations of salinity therefore seems to be unrelated to the missing Langmuir mixing in the climate model. The mechanisms that lead to the errors of salinity in the model are beyond the scope of this study.

\section{Summary}

In this study we have incorporated a third generation wave model, WAVEWATCH III, into CESM1.2 as an active component model. WAVEWATCH III is two-way coupled with the ocean model POP2 through a modified version of KPP to account for the effects of Langmuir mixing. The impact of Langmuir mixing on the global climate was first assessed in a Wave-Ocean coupled system. The Langmuir mixing is parameterized in KPP by applying an enhancement factor to the turbulent velocity scale with three different scalings following McWilliams and Sullivan (2000), Smyth et al. (2002) and Van Roekel et al. (2012), and subsequently considering the additional physics in the Van Roekel et al. (2012) scalings.

It is found that both MS2K and SS02 introduce too much mixing and overdeepen the MLD in CESM1.2. Though the modulation of the enhancement factor by stratification conditions in SS02 alleviates the over-deepening of MLD in the equatorial regions, it actually degrades in the extratropical regions.

The use of all of the VR12 scalings improves the simulation of both summer and winter MLD. Comparison of the aligned (VR12-AL) and misaligned cases (VR12-MA) shows modest differences in MLD in the Wave-Ocean only simulations. However, smaller RMSEs of MLD are observed in VR12-MA than in VR12-AL. VR12-MA may capture transient winds and waves better than VR12AL since considerably more physics and sensitivity have been included to agree with LES results of Harcourt and D'Asaro (2008) and Van Roekel et al. (2012), though the responses of the climate model may not appear to be significantly different.

Further estimating the enhanced entrainment at the base of ocean surface 
boundary layer (VR12-EN) gives prominent deepening of MLD compared with VR12-MA, highlighting the importance of enhanced entrainment by Langmuir mixing. However, the estimation of the Langmuir turbulence induced entrainment is only intended as an upper limit. A proper treatment of this effect requires more theoretical support.

The performance of CESM1.2 in terms of surface ocean mixing is improved by parameterizing Langmuir mixing in KPP, particularly when the effects of misaligned wind and waves and the penetration depth of Stokes drift are included. Specifically in the Wave-Ocean experiments,

- The shallow biases of MLD in the Southern Ocean are reduced by $27 \%$ in summer and $9 \%$ in winter;

- Ventilation is enhanced and the low concentration biases of pCFC-11 are reduced in the Southern Hemisphere, though significant low biases persist with a very similar pattern.

Preliminary fully coupled experiments confirm these improvements when other climate feedbacks are included, though at a reduced level. In addition, the cold biases in the subsurface ocean temperature are reduced when Langmuir turbulence is included, presumably through enhanced surface ocean mixing. Thus, it appears that climate model simulations can be improved by including a Langmuir mixing parameterization.

\section{Discussion and Conclusion}

Parameterizing Langmuir mixing in KPP through a Langmuir number related enhancement factor to the turbulent velocity scales highly depends on a LES based VKE scaling, which estimate the bulk effects of Langmuir turbulence on mixing within the ocean surface boundary layer. Our coupled Wave-Ocean experiments show that by switching from the MS2K scaling to the VR12 scaling alone, the unrealistic over-deepening of MLD in MS2K and SS02 could be eliminated. It is worth noting that the over-deepening of MLD by implementing 
MS2K and SS02 in the GFDL model (Fan and Griffies, 2014) are not as severe as shown here, suggesting some model dependence of parameterizing Langmuir mixing in this way.

Unsurprisingly, VR12-MA, in which the effects of the misaligned wind and waves and the penetration depth of Stokes drift are accounted for, improves the surface ocean mixing simulation in the climate model the most. However, it is interesting to note that as more and more physical processes are considered in the scaling (e.g. Harcourt and D'Asaro, 2008, Van Roekel et al., 2012), the resulting amount and distributions of the enhanced surface ocean mixing, and hence deepening in MLD, tend to converge. Newer and more complicated VKE scalings with different Langmuir numbers may be more accurate in the extreme cases, when approaching the upper and lower limits of the parameter space. However, the parameters in the climate model rarely approach those limits. This suggests that if no other new feedback mechanism is discovered, the climate model may be approaching diminishing returns for further improvements at greater computational cost in the VKE scaling. There is a large degree of consistency between our results with that of D'Asaro et al. (2014) in terms of the Langmuir turbulence induced deepening in MLD using a completely different parameterization of mixing (Harcourt, 2013), which further confirms this convergence.

Uncertainty still exists in how to best estimate the depth of the boundary layer, which is a key to capturing the entrainment fluxes. As we have seen in the comparisons among VR12-AL, VR12-MA and VR12-EN, enhanced entrainment introduces substantial potential deepening in MLD, more so than the effect due to misaligned wind and waves and Stokes drift penetration depth. The surface wave induced entrainment therefore requires more study and better representation. A possible upper limit estimate is given by VR12-EN.

Modest variations in the impact of Langmuir mixing shown here might result from using different source terms in WAVEWATCH III. In wave-only testing, a $17 \%$ greater average enhancement factor results from using Stokes drift generated by WAVEWATCH III using the Tolman and Chalikov (1996) source terms 
versus the Ardhuin et al. (2010) source terms, as the latter has $L a_{t} 20 \%$ larger on average for the same conditions. These enhancement factor discrepancies are small in comparison to the difference between the VR12-AL and VR12-MA parameterization forms shown in Figure $2 \mathrm{a}$, e versus Figure 2 $\mathrm{d}, \mathrm{h}$, and near the level of discrepancy between modeled and satellite-inferred Stokes drift magnitude (Webb and Fox-Kemper, 2011). The Ardhuin et al. (2010) source terms were chosen as they report better significant wave height in swell-dominated regions. This estimate predicts modest additional mixing in the climate model if the other source term formulation was chosen.

This work focused mainly on the influence of Langmuir mixing on the simulation of physical variables such as the MLD and ocean temperature. However, Langmuir deepening of MLD may have potential downstream impact on the biogeochemical modeling. The shallow winter MLD affects the formation of water masses (Weijer et al., 2012), which leads to weak mixing and ventilation of the intermediate waters. This may partly contribute to the biases in the simulated subsurface oxygen in CESM1(BGC) ${ }^{10}$ (Moore et al., 2013), and the low biases of the simulated total oceanic anthropogenic carbon inventory Wang et al., 2012, Long et al. 2013). The first hint of potential improvement in such errors were demonstrated here with CFCs. In addition, the shallow summer MLD may cause an underestimation of light limitation in a biogeochemistry model and affect the simulated oceanic carbon uptake by limiting the biological productivity (Moore et al. 2013, Rodgers et al., 2014). In a global ocean biogeochemistry model, Rodgers et al. (2014) found reduced uptake of $\mathrm{CO}_{2}$ by the Southern Ocean, and modified seasonal cycle of carbon and other ocean biogeochemical tracers, when wind stirring is parameterized and tuned to achieve best consistency with the observed summer MLD. Similar effects should result here as the summer MLD is improved through parameterizing Langmuir mixing, though quantitative estimation of these potential biogeochemical impacts remains outstanding.

${ }^{10}$ CESM, version 1 , with an ocean biogeochemistry module. 
This work represents a first step towards more comprehensive parameterizations of ocean surface gravity waves in the climate models. There are other wave related processes that require better representation. These include more than just the wind speed related influences of wind waves on the momentum flux, both for the atmosphere through a roughened ocean surface and for the the ocean through the redistribution of wind-driven momentum across the mixed layer (e.g., Sullivan et al. 2004, Garfinkel et al., 2011), the influences of wave induced bubbles on the tracer gas exchange at the air-sea interface and on the ocean surface buoyancy flux (Liang et al., 2011), and the interaction of Langmuir turbulence with the submesoscale eddies (Hamlington et al., 2014).

Parameterization of these processes will likely require parameters from a well simulated wave field in the climate model. However, an active wave model can be expensive. In our experiments, the model cost increased by $36 \%$ in the Wave-Ocean only experiments and $28 \%$ in the fully coupled experiments when Langmuir mixing was included in $\mathrm{KPP}^{11}$ Since the cost for WAVEWATCH III is constant with a specified grid resolution and coupling frequency (which determines the time step) and since increasing the wave model resolution does not affect Stokes drift significantly, one would expect this number to decrease when the low resolution WAVEWATCH III is coupled with a higher resolution ocean or atmosphere model. Nevertheless, a more efficient but accurate wave model is highly desirable for climate applications.

\footnotetext{
${ }^{11}$ The model cost in the Wave-Ocean only experiments are 3.02 and 4.09 pehours/simulated-year for CTRL and VR12-MA, respectively. With the nominal 3-degree resolution grid for POP2 and the G3F25T24 grid (see Table1 for details) for WAVEWATCH III; performed using 16 processors on Yellowstone at NCAR. The model cost in the fully coupled experiments are 40.12 and 51.35 pe-hours/simulated-year for CTRL and VR12-MA, respectively. Note that in the fully coupled experiments, the time step of WAVEWATCH III is one fourth of that in the Wave-Ocean coupled experiments.
} 


\section{Acknowledgments}

The authors would like to acknowledge fruitful conversations with Peter Sullivan and Jim McWilliams. Support for BFK, AW, QL from NSF 1258907, 0934737 and NASA ROSES NNX09AF38G is gratefully acknowledged. We would also like to acknowledge high-performance computing support from Yellowstone (ark:/85065/d7wd3xhc) provided by NCAR's Computational and Information Systems Laboratory, sponsored by the National Science Foundation. NCAR is sponsored by the National Science Foundation. We also appreciate the very helpful comments and suggestions from the editor and the three anonymous reviewers.

\section{References}

Abernathey, R., Marshall, J., Mazloff, M., 2015. Critical-layer enhancement of mesoscale eddy stirring in the southern ocean. Submitted to Journal of Physical Oceanography.

Ardhuin, F., Rogers, E., Babanin, A. V., Filipot, J.-F., Magne, R., Roland, A., van der Westhuysen, A., Queffeulou, P., Lefevre, J.-M., Aouf, L., Collard, F., 2010. Semiempirical dissipation source functions for ocean waves. Part I: Definition, calibration, and validation. Journal of Physical Oceanography 40, 1917-1941.

Belcher, S. E., Grant, A. L. M., Hanley, K. E., Fox-kemper, B., Roekel, L. V., Sullivan, P. P., Large, W. G., Brown, A., Hines, A., Calvert, D., Rutgersson, A., Pettersson, H., Bidlot, J.-r., Janssen, P. A. E. M., Polton, J. A., 2012. A global perspective on Langmuir turbulence in the ocean surface boundary layer. Geophysical Research Letters 39, L18605.

Cavaleri, L., Fox-Kemper, B., Hemer, M., 2012. Wind waves in the coupled climate system. Bulletin of the American Meteorological Society 93, 16511661. 
Craig, A. P., Vertenstein, M., Jacob, R., 2011. A new flexible coupler for earth system modeling developed for CCSM4 and CESM1. International Journal of High Performance Computing Applications 26, 31-42.

Danabasoglu, G., Bates, S. C., Briegleb, B. P., Jayne, S. R., Jochum, M., Large, W. G., Peacock, S., Yeager, S. G., 2012. The CCSM4 Ocean Component. Journal of Climate 25, 1361-1389.

Danabasoglu, G., Yeager, S. G., Bailey, D., Behrens, E., Bentsen, M., Bi, D., Biastoch, A., Böning, C., Bozec, A., Canuto, V. M., Cassou, C., Chassignet, E., Coward, A. C., Danilov, S., Diansky, N., Drange, H., Farneti, R., Fernandez, E., Fogli, P. G., Forget, G., Fujii, Y., Griffies, S. M., Gusev, A., Heimbach, P., Howard, A., Jung, T., Kelley, M., Large, W. G., Leboissetier, A., Lu, J., Madec, G., Marsland, S. J., Masina, S., Navarra, A., a.J. George Nurser, Pirani, A., y Mélia, D. S., Samuels, B. L., Scheinert, M., Sidorenko, D., Treguier, A.-M., Tsujino, H., Uotila, P., Valcke, S., Voldoire, A., Wang, Q., 2014. North Atlantic simulations in Coordinated Ocean-ice Reference Experiments phase II (CORE-II). Part I: Mean states. Ocean Modelling 73, 76-107.

D'Asaro, E. A., Thomson, J., Shcherbina, A. Y., Harcourt, R. R., Cronin, M. F., Hemer, M. A., Fox-Kemper, B., 2014. Quantifying upper ocean turbulence driven by surface waves. Geophysical Research Letters 41, 102-107.

de Boyer Montégut, C., Madec, C., Fischer, A. S., Lazar, A., Iudicone, D., 2004. Mixed layer depth over the global ocean: An examination of profile data and a profile-based climatology. Journal of Geophysical Research 109, C12003.

Fan, Y., Griffies, S. M., 2014. Impacts of parameterized langmuir turbulence and nonbreaking wave mixing in global climate simulations. Journal of Climate $27,4752-4775$.

Fox-Kemper, B., Danabasoglu, G., Ferrari, R., Griffies, S. M., Hallberg, R. W., Holland, M., Maltrud, M., Peacock, S., Samuels, B., 2011. Parameterization of mixed layer eddies. III: Implementation and impact in global ocean climate simulations. Ocean Modelling 39, 61-78. 
Fox-Kemper, B., Ferrari, R., Hallberg, R. W., 2008. Parameterization of mixed layer eddies. Part I: Theory and diagnosis. Journal of Physical Oceanography $38,1145-1165$.

Fox-Kemper, B., Lumpkin, R., Bryan, F. O., 2013. Lateral transport in the ocean interior. In: Siedler, G., Griffies, S. M., Gould, J., Church, J. A. (Eds.), Ocean Circulation and Climate: A 21st century perspective. Vol. 103 of International Geophysics Series. Academic Press (Elsevier Online), Ch. 8, pp. $185-209$.

URL http://dx.doi.org/10.1016/B978-0-12-391851-2.00008-8

Garfinkel, C. I., Molod, a. M., Oman, L. D., Song, I.-S., 2011. Improvement of the GEOS-5 AGCM upon updating the air-sea roughness parameterization. Geophysical Research Letters 38, L18702.

Gent, P. R., Danabasoglu, G., Donner, L. J., Holland, M. M., Hunke, E. C., Jayne, S. R., Lawrence, D. M., Neale, R. B., Rasch, P. J., Vertenstein, M., Worley, P. H., Yang, Z.-L., Zhang, M., 2011. The Community Climate System Model Version 4. Journal of Climate 24, 4973-4991.

Grant, A. L. M., Belcher, S. E., 2009. Characteristics of Langmuir turbulence in the ocean mixed layer. Journal of Physical Oceanography 39, 1871-1887. URL http://journals . ametsoc .org/doi/abs/10.1175/2009JP04119.1

Hamlington, P. E., Van Roekel, L. P., Fox-Kemper, B., Julien, K., Chini, G. P., 2014. Langmuirsubmesoscale interactions: Descriptive analysis of multiscale frontal spindown simulations. Journal of Physical Oceanography 44, 2249 2272 .

Haney, S., Fox-Kemper, B., Julien, K., 2014. Instabilities in the wave forced ocean mixed layer. In preparation.

Hanley, K. E., Belcher, S. E., Sullivan, P. P., 2010. A global climatology of windwave interaction. Journal of Physical Oceanography 40, 1263-1282. 
Harcourt, R. R., 2013. A second-moment closure model of Langmuir turbulence. Journal of Physical Oceanography 43, 673-697.

Harcourt, R. R., D'Asaro, E. A., 2008. Large-eddy simulation of Langmuir turbulence in pure wind seas. Journal of Physical Oceanography 38, 15421562.

Jochum, M., Briegleb, B. P., Danabasoglu, G., Large, W. G., Norton, N. J., Jayne, S. R., Alford, M. H., Bryan, F. O., 2013. The impact of oceanic nearinertial waves on climate. Journal of Climate 26, 2833-2844.

Kantha, L. H., Clayson, C. A., 2004. On the effect of surface gravity waves on mixing in the oceanic mixed layer. Ocean Modelling 6, 101-124.

Key, R. M., Kozyr, A., Sabine, C. L., Lee, K., Wanninkhof, R., Bullister, J. L., Feely, R. a., Millero, F. J., Mordy, C., Peng, T.-H., 2004. A global ocean carbon climatology: Results from Global Data Analysis Project (GLODAP). Global Biogeochemical Cycles 18, GB4031.

Kirtman, B. P., Schneider, E. K., Straus, D. M., Min, D., Burgman, R., 2011. How weather impacts the forced climate response. Climate Dynamics 37, 2389-2416.

Langmuir, I., 1938. Surface motion of water induced by wind. Science 87, 119123.

Large, W. G., Danabasoglu, G., Doney, S. C., 1997. Sensitivity to surface forcing and boundary layer mixing in a global ocean model: Annual-mean climatology. Journal of Physical Oceanography 27, 2418-2447.

Large, W. G., Mcwilliams, J. C., Doney, S. C., 1994. Oceanic vertical mixing: A review and a model with a nonlocal boundary layer parameterization. Reviews of Geophysics 32, 363-403.

Large, W. G., Yeager, S. G., 2009. The global climatology of an interannually varying airsea flux data set. Climate Dynamics 33, 341-364. 
Levitus, S., Boyer, T., Concright, M., Johnson, D., OBrien, T., Antonov, J., Stephens, C., Garfield, R., 1998. Introduction. Vol. I, World Ocean Database, NOAA Atlas NESDIS18, 346.

Li, M., Garrett, C., 1997. Mixed layer deepening due to Langmuir circulation. Journal of Physical Oceanography 27, 121-132.

Liang, J.-H., McWilliams, J. C., Sullivan, P. P., Baschek, B., 2011. Modeling bubbles and dissolved gases in the ocean. Journal of Geophysical Research 116, C03015.

Long, M. C., Lindsay, K., Peacock, S., Moore, J. K., Doney, S. C., 2013. Twentieth-century oceanic carbon uptake and storage in CESM1(BGC)*. Journal of Climate 26, 6775-6800.

URL http://journals.ametsoc.org/doi/abs/10.1175/ JCLI-D-12-00184.1

McClean, J. L., Bader, D. C., Bryan, F. O., Maltrud, M. E., Dennis, J. M., Mirin, A. a., Jones, P. W., Kim, Y. Y., Ivanova, D. P., Vertenstein, M., Boyle, J. S., Jacob, R. L., Norton, N., Craig, A., Worley, P. H., 2011. A prototype two-decade fully-coupled fine-resolution CCSM simulation. Ocean Modelling 39, 10-30.

McWilliams, J. C., Fox-Kemper, B., 2013. Oceanic wave-balanced surface fronts and filaments. Journal of Fluid Mechanics 730, 464-490.

McWilliams, J. C., Huckle, E., Liang, J., Sullivan, P. P., 2014. Langmuir turbulence in swell. Journal of Physical Oceanography 44, 870-890.

URL http: //journals . ametsoc .org/doi/abs/10.1175/JPO-D-13-0122.1

McWilliams, J. C., Sullivan, P. P., 2000. Vertical mixing by Langmuir circulations. Spill Science and Technology Bulletin 6, 225-237.

McWilliams, J. C., Sullivan, P. P., Moeng, C.-H., 1997. Langmuir turbulence in the ocean. Journal of Fluid Mechanics 334, 1-30. 
Moore, J. K., Lindsay, K., Doney, S. C., Long, M. C., Misumi, K., 2013. Marine ecosystem dynamics and biogeochemical cycling in the community earth system model [CESM1(BGC)]: Comparison of the 1990s with the 2090s under the RCP4.5 and RCP8.5 scenarios. Journal of Climate 26, 9291-9312.

Noh, Y., Lee, W. S., 2008. Mixed and mixing layer depths simulated by an OGCM. Journal of Oceanography 64, 217-225.

Rascle, N., Ardhuin, F., Queffeulou, P., Croizé-Fillon, D., 2008. A global wave parameter database for geophysical applications. Part 1: Wavecurrentturbulence interaction parameters for the open ocean based on traditional parameterizations. Ocean Modelling 25, 154-171.

Reckinger, S. J., Fox-Kemper, B., 2015. Anisotropy in mesoscale eddy transport. I: Global sensitivity. In preparation.

Rodgers, K. B., Aumont, O., Mikaloff Fletcher, S. E., Plancherel, Y., Bopp, L., De Boyer Montégut, C., Iudicone, D., Keeling, R. F., Madec, G., Wanninkhof, R., 2014. Strong sensitivity of Southern Ocean carbon uptake and nutrient cycling to wind stirring. Biogeosciences 11, 4077-4098.

Sallée, J.-B., Shuckburgh, E., Bruneau, N., Meijers, a. J. S., Bracegirdle, T. J., Wang, Z., 2013. Assessment of Southern Ocean mixed-layer depths in CMIP5 models: Historical bias and forcing response. Journal of Geophysical Research: Oceans 118, 1845-1862.

Smith, R. S., Jones, P. W., Briegleb, B., Bryan, F., Danabasoglu, G., Dennis, J., Dukowicz, J., Eden, C., Fox-Kemper, B., Gent, P.Smith, Smith, R. S., Jones, P. W. S., Hecht, M., Jayne, S., Jochum, M., Large, W., Lindsay, K., Maltrud, M., Norton, N., Peacock, S., Vertenstein, M., Yeager, S., 2010. The Parallel Ocean Program (POP) reference manual. Tech. Rep. LAUR-10-01853, Los Alamos National Laboratory.

Smyth, W. D., Skyllingstad, E. D., Crawford, G. B., Wijesekera, H., 2002. 
Nonlocal fluxes and Stokes drift effects in the K-profile parameterization. Ocean Dynamics 52, 104-115.

Steele, M., Morley, R., Ermold, W., 2001. PHC: A global ocean hydrography with a high-quality Arctic Ocean. Journal of Climate 14, 2079-2087.

Sullivan, P. P., McWilliams, J. C., 2010. Dynamics of winds and currents coupled to surface waves. Annual Review of Fluid Mechanics 42, 19-42.

Sullivan, P. P., McWILLIAMS, J. C., Melville, W. K., 2004. The oceanic boundary layer driven by wave breaking with stochastic variability. Part 1 . Direct numerical simulations. Journal of Fluid Mechanics 507, 143-174.

Sutherland, G., Christensen, K. H., Ward, B., 2014a. Evaluating Langmuir turbulence parameterizations in the ocean surface boundary layer. Journal of Geophysical Research: Oceans 119, 1899-1910.

Sutherland, G., Reverdin, G., Marié, L., Ward, B., 2014b. Mixed and mixing layer depths in the ocean surface boundary layer under conditions of diurnal stratification. Geophysical Research Letters 41, 8469-8476.

URL http://doi .wiley · com/10.1002/2014GL061939

Thorpe, S. A., 2007. An Introduction to Ocean Turbulence. Cambridge University Press, New York.

Tolman, H. L., 2009. User manual and system documentation of WAVEWATCH III version 3.14. Tech. Rep. 276, NOAA / NWS / NCEP / MMAB.

Tolman, H. L., Chalikov, D., 1996. Source terms in a third-generation wind wave model. Journal of Physical Oceanography 26, 2497-2518.

Van Roekel, L. P., Fox-Kemper, B., Sullivan, P. P., Hamlington, P. E., Haney, S. R., 2012. The form and orientation of Langmuir cells for misaligned winds and waves. Journal of Geophysical Research 117, C05001. 
Vertenstein, M., Bertini, A., Craig, T., Edwards, J., Mai, A., Schollenberger, J., 2013. CESM users guide (CESM1.2 release series). Tech. rep., The National Center for Atmosphere Research.

Wang, S., Moore, J. K., Primeau, F. W., Khatiwala, S., 2012. Simulation of anthropogenic CO 2 uptake in the CCSM3.1 ocean circulation-biogeochemical model: Comparison with data-based estimates. Biogeosciences 9, 1321-1336.

Webb, A., 2013. Stokes Drift and Meshless Wave Modeling. Ph.D. thesis, University of Colorado.

Webb, A., Fox-Kemper, B., 2011. Wave spectral moments and Stokes drift estimation. Ocean Modelling 40, 273-288.

Webb, A., Fox-Kemper, B., 2015. Impacts of wave spreading and multidirectional waves on estimating Stokes drift. Ocean Modelling.

URL http://dx.doi.org/10.1016/j.ocemod.2014.12.007

Weijer, W., Sloyan, B. M., Maltrud, M. E., Jeffery, N., Hecht, M. W., Hartin, C. a., van Sebille, E., Wainer, I., Landrum, L., 2012. The Southern Ocean and its climate in CCSM4. Journal of Climate 25, 2652-2675. 


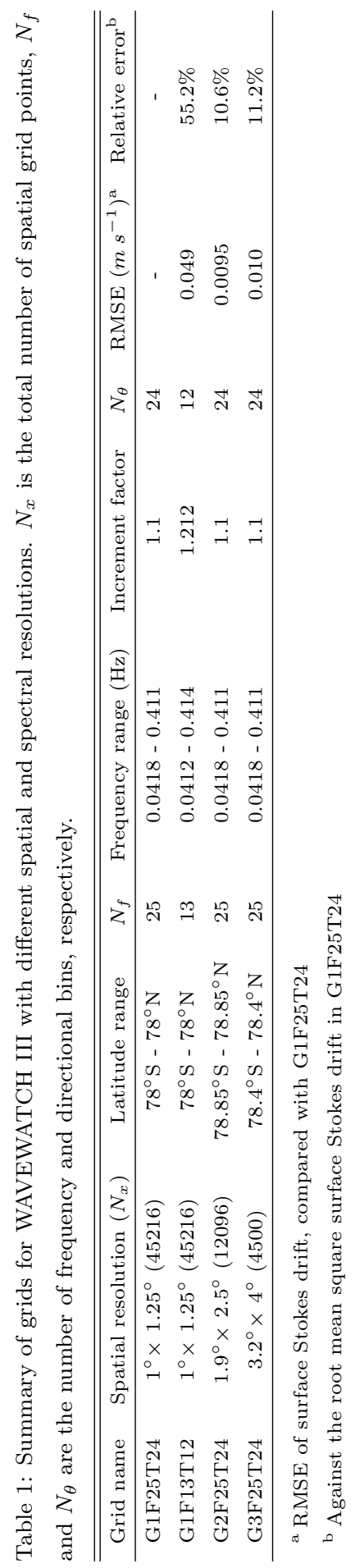




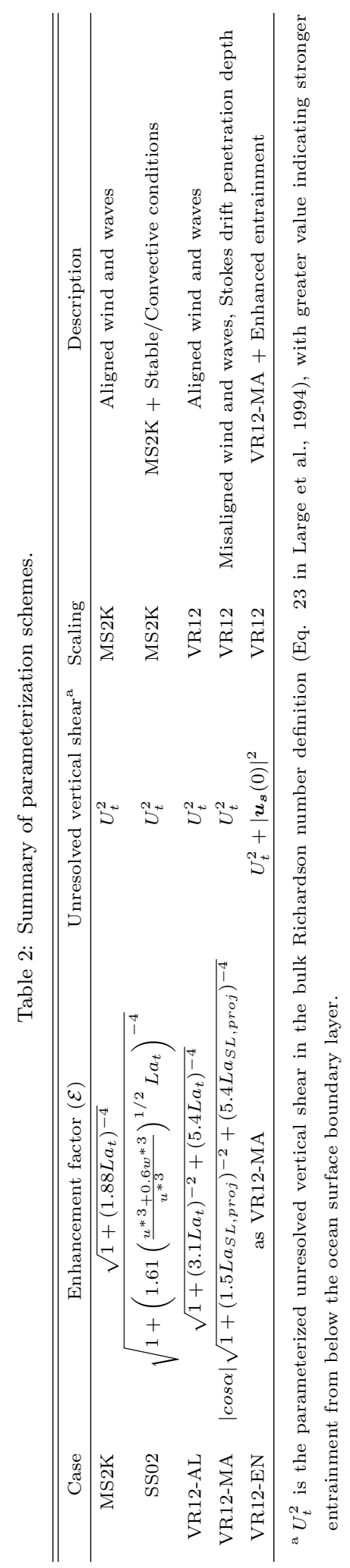




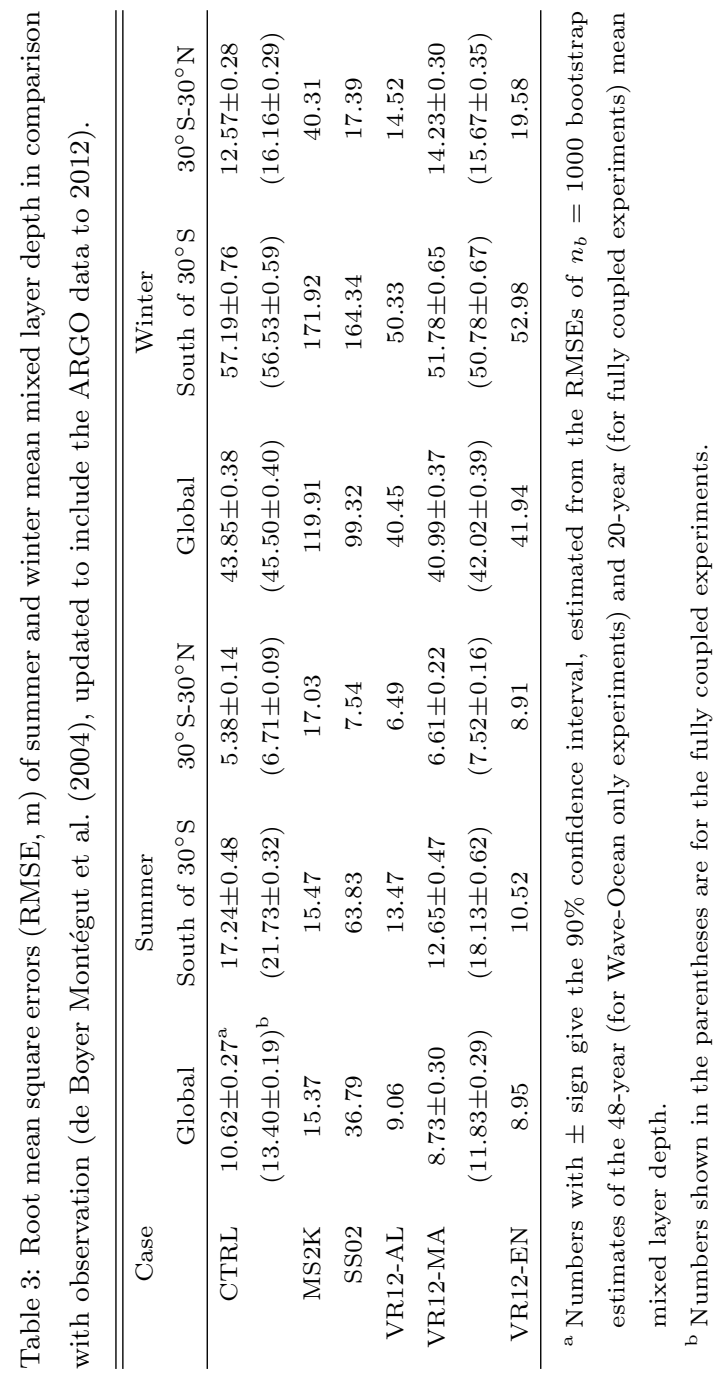


Table 4: Root mean square difference (patm) of zonal averaged pCFC-11 compared with observation (GLODAP; Key et al. (2004)).

\begin{tabular}{rcc}
\hline \hline Case & Global & Southern Hemisphere \\
\hline CTRL & 23.90 & 20.20 \\
MS2K & 29.89 & 30.99 \\
SS02 & 34.16 & 41.90 \\
VR12-AL & 22.14 & 18.53 \\
VR12-MA & 23.23 & 18.90 \\
VR12-EN & 20.67 & 16.44 \\
\hline
\end{tabular}


Table 5: Root mean square errors (RMSE, ${ }^{\circ} \mathrm{C}$ ) of the 20-year averaged ocean temperature at the surface and at the depth of $100 \mathrm{~m}$ in the fully coupled experiment, compared with observation (PHC3, Steele et al. 2001).

\begin{tabular}{rcccc}
\hline \hline Case (depth) & Global & $90^{\circ} \mathrm{S}-30^{\circ} \mathrm{S}$ & $30^{\circ} \mathrm{S}-30^{\circ} \mathrm{N}$ & $30^{\circ} \mathrm{N}-90^{\circ} \mathrm{N}$ \\
\hline CTRL (0 m) & $1.53 \pm 0.02^{\mathrm{a}}$ & $0.90 \pm 0.02$ & $1.10 \pm 0.02$ & $3.01 \pm 0.05$ \\
VR12-MA (0 m) & $1.54 \pm 0.01$ & $1.04 \pm 0.03$ & $1.14 \pm 0.01$ & $2.90 \pm 0.04$ \\
CTRL (100 m) & $1.96 \pm 0.02$ & $1.39 \pm 0.01$ & $1.92 \pm 0.02$ & $2.88 \pm 0.04$ \\
VR12-MA (100 m) & $1.75 \pm 0.02$ & $1.29 \pm 0.01$ & $1.60 \pm 0.02$ & $2.76 \pm 0.04$ \\
\hline
\end{tabular}

${ }^{\text {a }}$ Numbers with \pm sign give the $90 \%$ confidence interval, estimated from the RMSEs of $n_{b}=1000$ bootstrap estimates of the 20-year mean surface and subsurface temperature. 


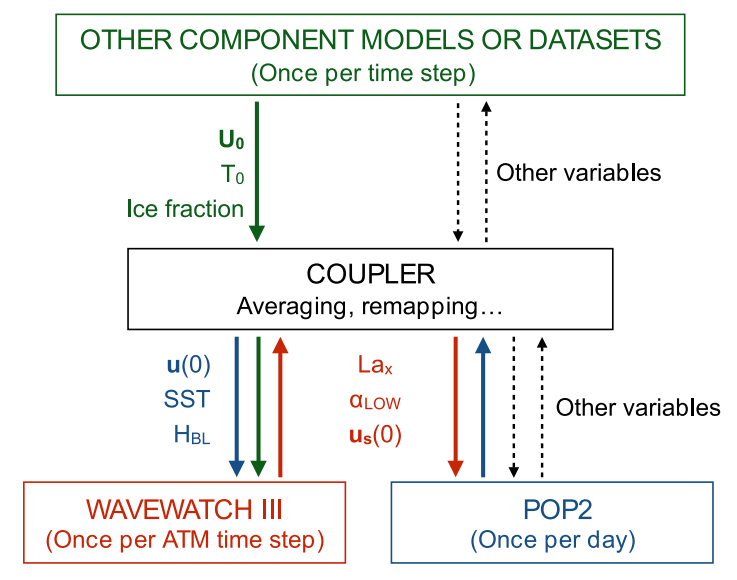

Figure 1: Schematic diagram of WAVEWATCH III coupling in CESM1.2. The arrows in color show the variable flows passing between WAVEWATCH III and other model components, with $\boldsymbol{U}_{\mathbf{0}}$ and $T_{0}$ the surface wind and temperature in the atmosphere; $\boldsymbol{u}(0)$ the surface current in the ocean; $H_{B L}$ the ocean surface boundary layer depth; $L a_{x}$ the Langmuir number ( $L a_{t}$ or $\left.L a_{S L, \text { proj }}\right) ; \alpha_{L O W}$ the angle between the wind and Langmuir cells and $\boldsymbol{u}_{\boldsymbol{s}}(0)$ the surface Stokes drift. The black dash arrows indicate all other variable flows passing between the ocean component and other model components. The coupling frequency is labeled in parentheses for each model component. Note the different coupling frequencies of wave-atmosphere coupling and wave-ocean coupling. See text for more detail. 

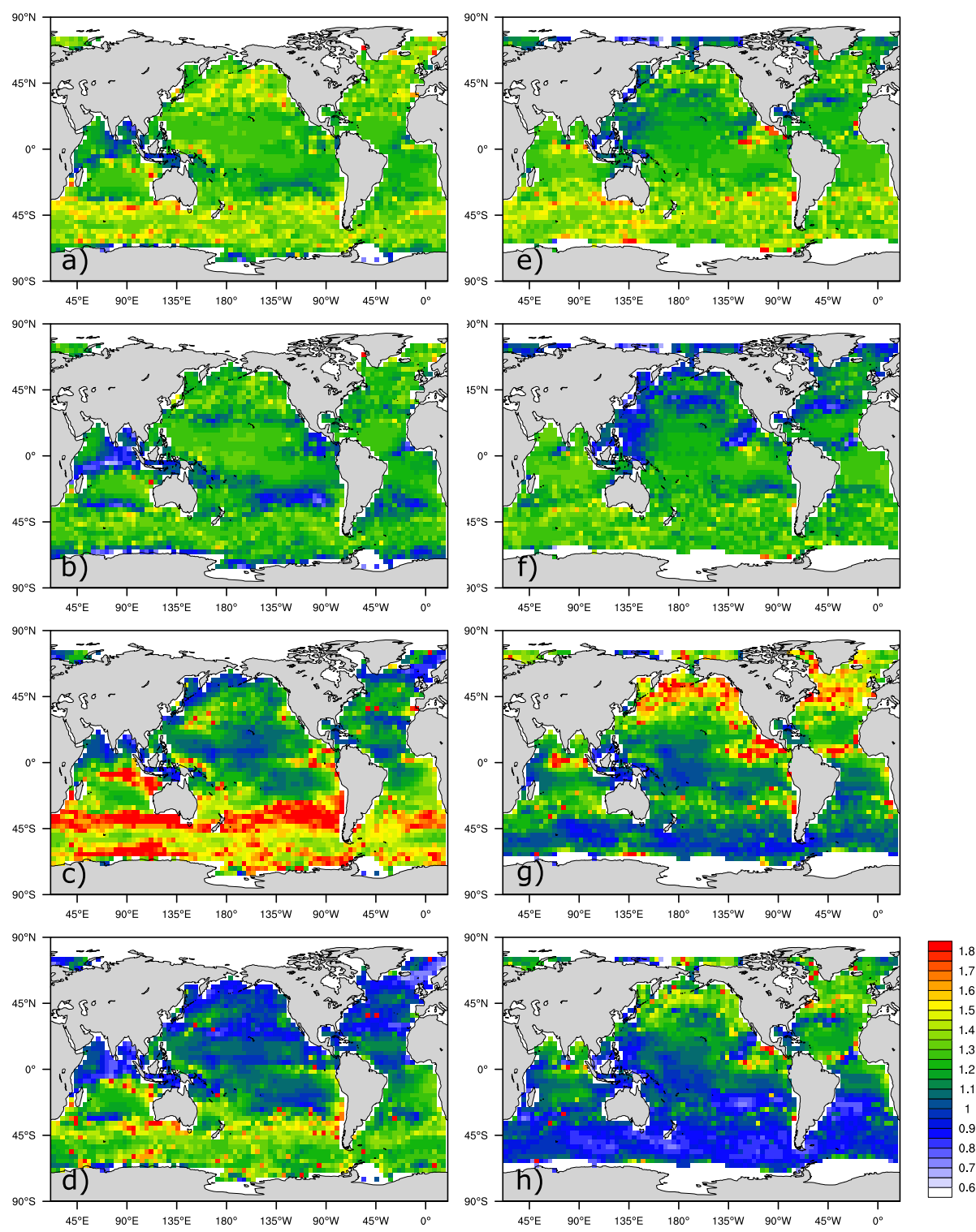

Figure 2: The mixed layer averaged vertical turbulent kinetic energy (VKE) normalized by the friction velocity squared, predicted from the scalings with different Langmuir numbers, showing the effects of misaligned wind and waves and Stokes drift penetration depth. The normalized VKEs are calculated from scalings with (a)(e) $L a_{t}$; (b)(f) $L a_{\text {proj }}$; (c)(g) $L a_{S L}$ and (d)(h) $L a_{S L, p r o j}$, following Van Roekel et al. (2012), averaged over Jan. (a) - (d) and Jul. (e) - (h) of the model year 247. In the limit of no waves $(L a \rightarrow \infty)$, the normalized VKE is 0.6, determined from a shear turbulence only simulation (Van Roekel et al., 2012). Note that (a) (e) and (d)(h) are corresponding to experiment VR12-AL and VR12-MA, respectively. 

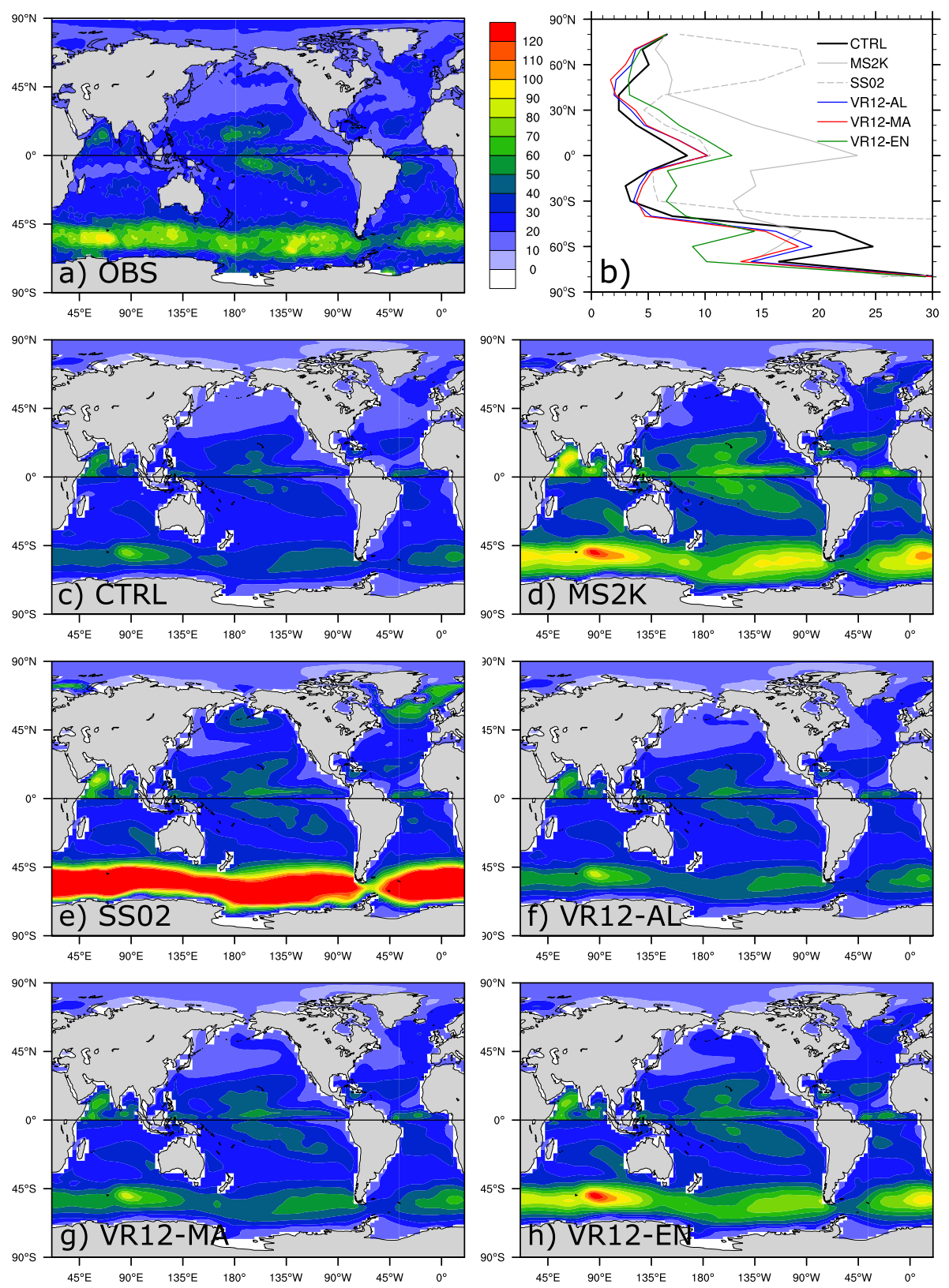

Figure 3: Impact of Langmuir mixing on the summer mean mixed layer depth (MLD; $\mathrm{m}$ ) for both hemispheres. (a) shows the observation from de Boyer Montégut et al. (2004), updated to include the ARGO data to 2012. (c) shows the control run without Langmuir mixing. (d) (h) are results with Langmuir mixing implemented in different parameterization schemes (See Table 2 for description). MLDs are averaged over Jul., Aug. and Sep. (JAS) for the Northern Hemisphere (NH) and Jan., Feb. and Mar. (JFM) for the Southern Hemisphere (SH). (b) shows the latitudinal distribution of root mean square errors. 

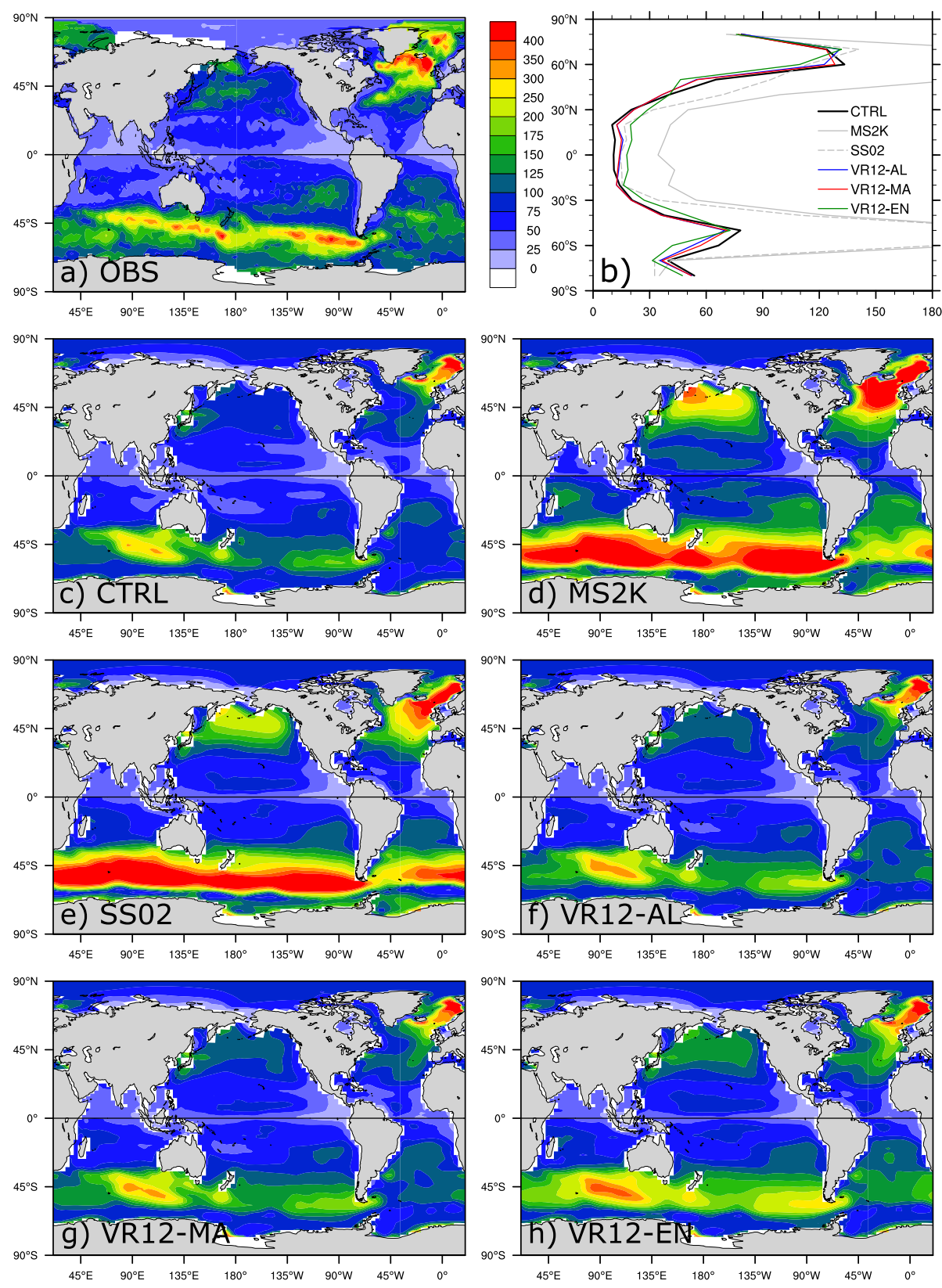

Figure 4: As Fig. 3 but for the winter mixed layer depth. Averaged over JFM for the NH and JAS for the SH. 

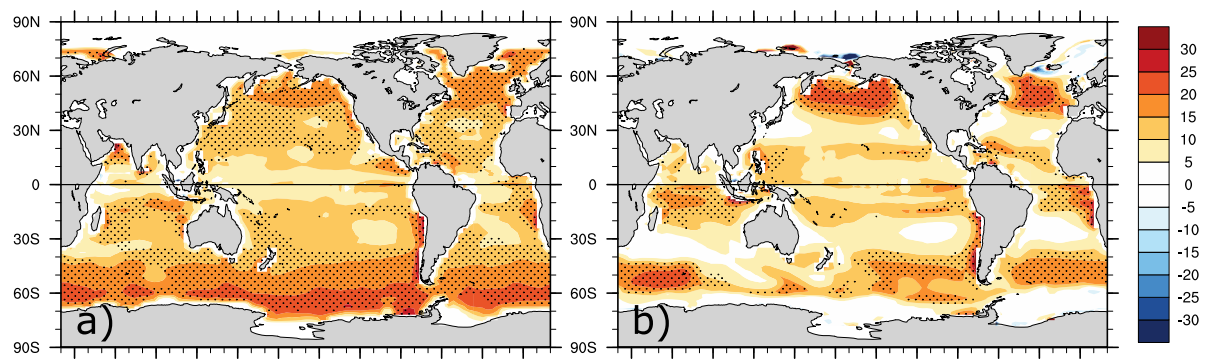

30E GOE 90E 120E 150E 180 150W120W 90W 60W 30W 0

30E GOE 90E 120E 150E 180 150W120W 90W 6OW 30W 0

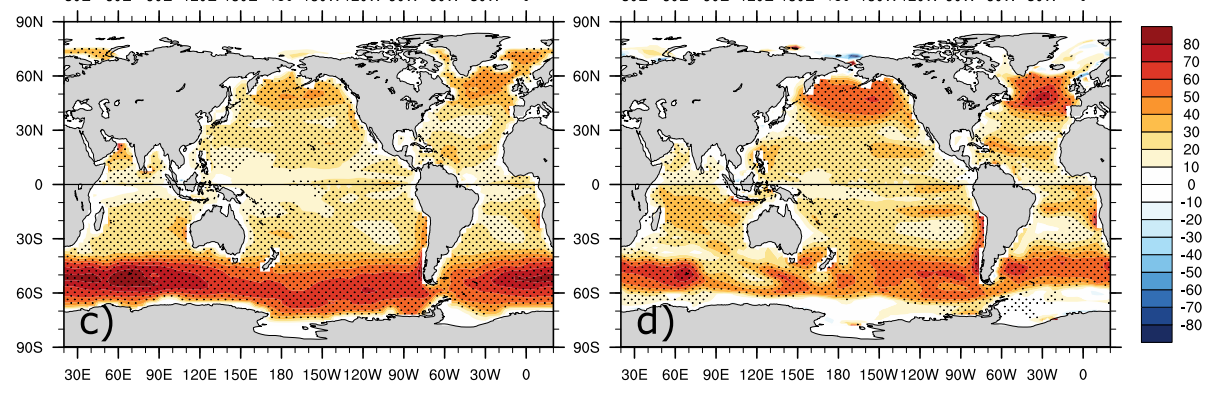

Figure 5: Langmuir mixing induced (a)(c) summer and (b)(d) winter mean mixed layer depth (MLD) deepening in (a)(b) VR12-MA and (c)(d) VR12-EN, shown by the percentage change (\%) in comparison with CTRL. Regions where MLD changes are significant (i.e. greater than the MLD standard deviation based on monthly data in CTRL) are shaded by dots. 

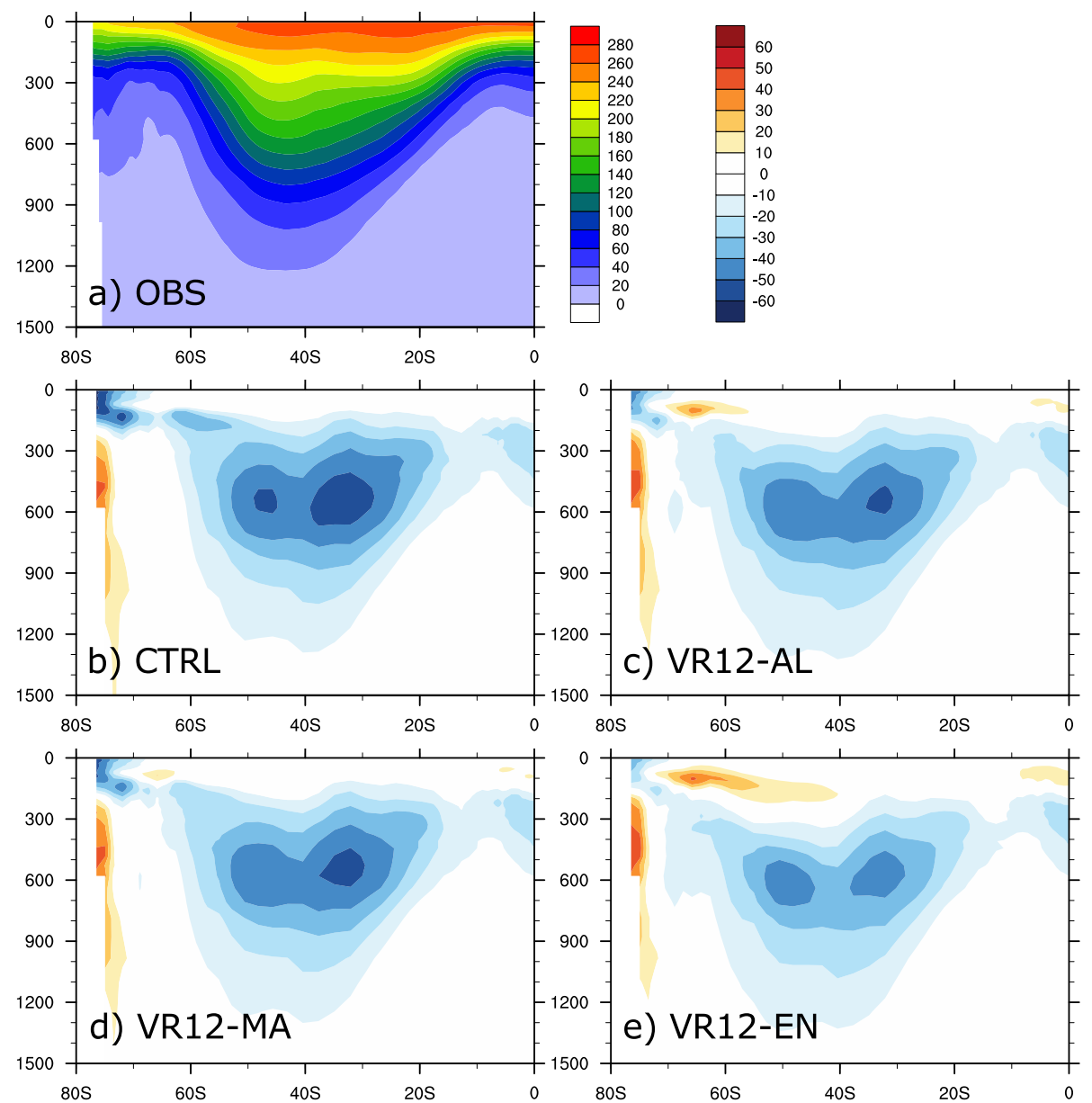

Figure 6: Impact of Langmuir mixing on the zonal mean pCFC-11 (patm) in the Southern Hemisphere. (a) shows the observation (Key et al. 2004). (b) - (e) show the anomaly from the observation for the cases CTRL, VR12-AL, VR12-MA and VR12-EN, respectively. Note the continuous reduction of low concentration biases as more physics are included and the emergence of high concentration biases at the surface in VR12-AL and VR12-EN. 

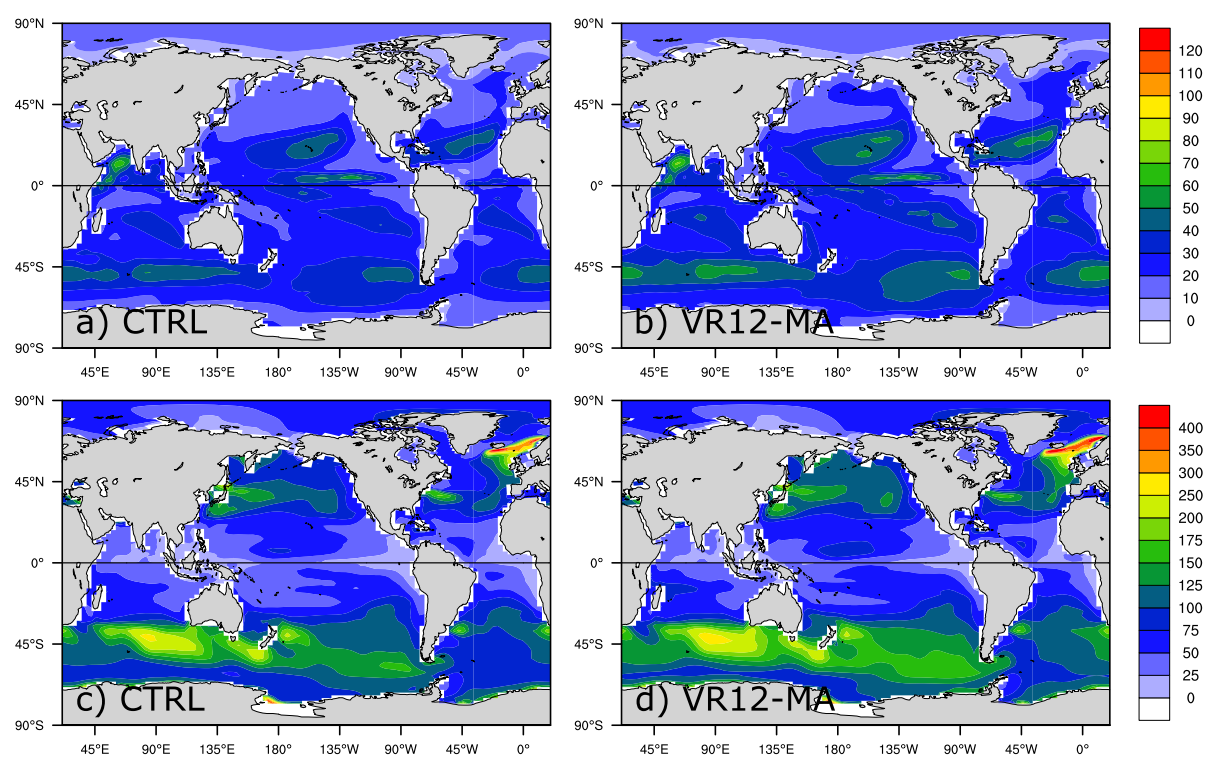

Figure 7: As Fig. 3 and Fig. 4 but for the fully coupled experiments. (a)(b) Summer and (c)(d) winter mean mixed layer depth (m) for experiments: (a)(c) without Langmuir mixing $(\mathrm{CTRL}) ;(\mathrm{b})(\mathrm{d})$ with Langmuir mixing accounting for the misaligned wind and waves and the Stokes drift penetration depth (VR12-MA). 

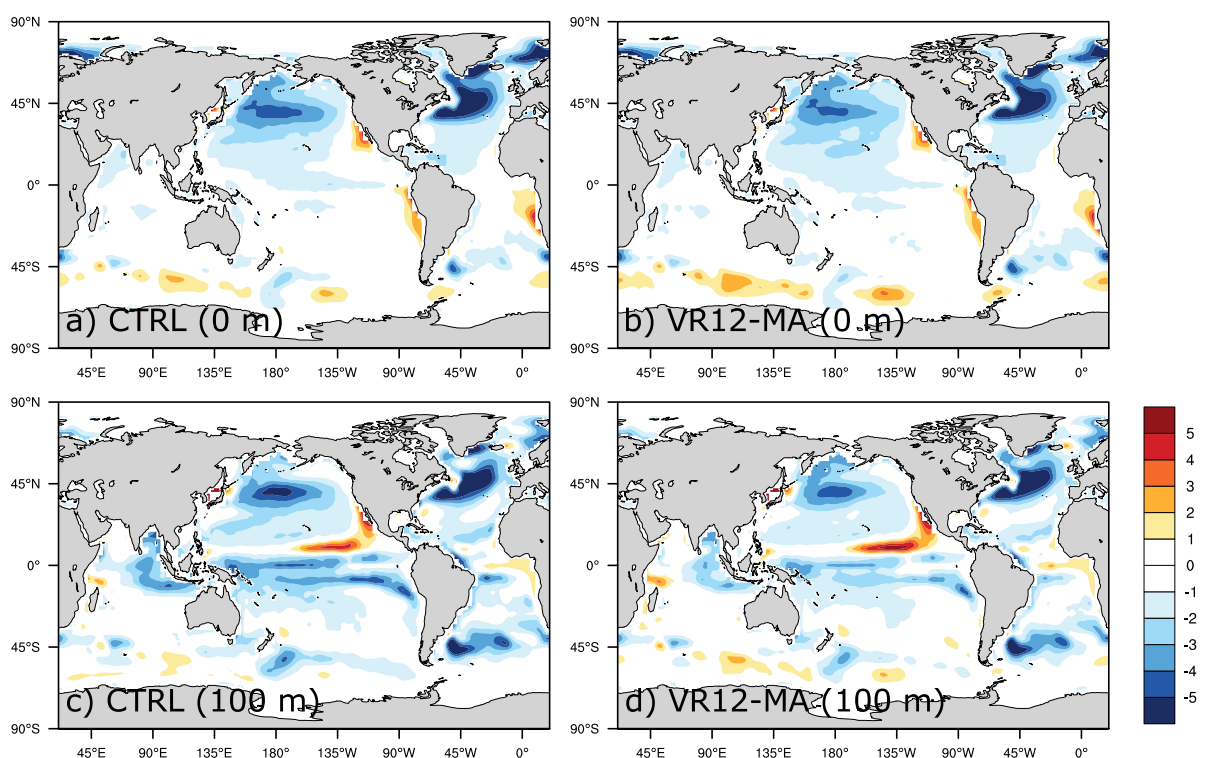

Figure 8: Maps of errors in the simulations of annual mean SST (upper panels) and ocean temperature at depth of $100 \mathrm{~m}$ (lower panels) in CTRL and VR12-MA. The errors are computed as model minus observation, where the observations are from the PHC3 dataset. 


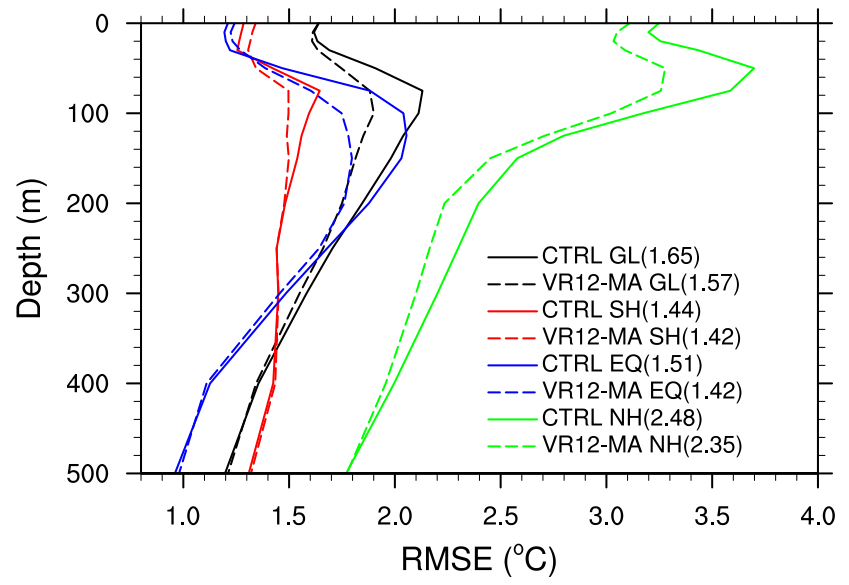

Figure 9: RMSEs of temperature versus depth. RMSEs are computed over the global ocean (black), south of $30^{\circ} \mathrm{S}$ (red), $30^{\circ} \mathrm{S}$ to $30^{\circ} \mathrm{N}$ (blue) and north of $30^{\circ} \mathrm{N}$ (green) for CTRL (line) and VR12-MA (dash). Numbers in parentheses are total RMSEs over the upper $500 \mathrm{~m}$. The monthly mean ocean temperature in both experiments are compared against the PHC3 monthly climatology. 